\title{
Local Cooling, Plasma Reheating and Thermal Pinching Induced by Single Aerosol Droplets Injected into an Inductively Coupled Plasma
}

\author{
George C.-Y. Chan ${ }^{1,2 *}$ and Gary M. Hieftje ${ }^{1}$ \\ ${ }^{1 .}$ Department of Chemistry, Indiana University, 800 E. Kirkwood Avenue, Bloomington, IN 47405, USA. \\ 2. Lawrence Berkeley National Laboratory, 1 Cyclotron Road, Berkeley, CA 94720, USA.
}




\section{Abstract}

The injection of a single micrometer-sized droplet into an analytical inductively coupled plasma (ICP) perturbs the plasma and involves three sequential effects: local cooling, thermal pinching and plasma reheating. Time-resolved two-dimensional monochromatic imaging of the load-coil region of an ICP was used to monitor this sequence of plasma perturbations. When a microdroplet enters the plasma, it acts as a local heat sink and cools the nearby plasma region. The cooling effect is considered local, although the cooling volume can be large and extends $6 \mathrm{~mm}$ from the physical location of the vaporizing droplet. The liberated hydrogen, from decomposition of water, causes a thermal pinch effect by increasing the thermal conductivity of the bulk plasma and accelerating heat loss at the plasma periphery. As a response to the heat loss, the plasma shrinks in size, which increases its power density. Plasma shrinkage starts around the same time when the microdroplet enters the plasma and lasts at least $2 \mathrm{~ms}$ after the droplet leaves the load-coil region. Once the vaporizing droplet passes through a particular plasma volume, that volume is reheated to an even higher temperature than under steady-state conditions. Because of the opposing effects of plasma cooling and reheating, the plasma conditions are different upstream (downward) and downstream (upward) from a vaporizing droplet - cooling dominates the downstream region whereas reheating controls in the upstream domain. The boundary between the local cooling and reheating zones is sharp and is only $\sim 1 \mathrm{~mm}$ thick. The reheating effect persists a relatively long time in the plasma, at least up to $4 \mathrm{~ms}$ after the droplet moves out of the load-coil region. The restoration of plasma equilibrium after the perturbation induced by microdroplet injection is slow. Microdroplet injection also induces a momentary change 
in plasma impedance, and the impedance change was found to correlate qualitatively with the different stages of plasma perturbation.

Keywords: inductively coupled plasma-atomic emission spectrometry; monodisperse droplets; local cooling; plasma pinch; plasma impedance 


\section{Introduction}

Recent advances in single-particle inductively coupled plasma (sp-ICP) [1] spectrometry enable the elemental and isotopic measurements of individual entities (e.g., nanoparticles [2, 3] and biological cells [4, 5]), and provide information such as particle-size distributions [2] and their stoichiometry [3] that are otherwise unavailable from simple bulk chemical analyses. One approach for sp-ICP measurement is to embed the particle inside a solution droplet and introduce this droplet into the ICP. Ideally, each such droplet contains no more than a single particle, and the analytical signals from individual entities are well separated in time and can be registered in the form of discrete signal spikes.

Droplet introduction for sp-ICP spectrometry can be either through the use of a conventional pneumatic nebulizer, which generates aerosols with a range of sizes, or a specialized device that dispenses microdroplets with a nominally monodisperse size distribution. Analytical characteristics and performance of these two sample-introduction approaches for sp-ICP spectrometry have been evaluated by Franze et al. [6] and Gschwind et al. [7]. Compared to a conventional pneumatic nebulizer, a monodisperse microdroplet dispenser offers important advantages. One such advantage is that the solution can be doped with the analyte of interest, and simultaneous measurement of the solution signal together with that from the particle provides a means of particle-mass calibration. Because of the monodisperse size distribution of the droplets, the doped analyte mass is constant from droplet to droplet and can be readily calculated from the concentration of the standard solution and the measured droplet size. The Niemax 
group [2] established this calibration approach and successfully demonstrated silica- and gold-nanoparticle analyses with calibration from simple aqueous standards. Another advantage is that, again because of the monodisperse droplet size, the time (which also translates into height in the plasma) required for the droplet to undergo complete desolvation, atomization, excitation and ionization is very reproducible from droplet to droplet. This unique feature makes the arrival time of the signal spike predictable and the optimization for detection volume in the plasma straightforward.

The reproducible timing and trajectory of monodisperse microdroplets allow researchers to closely follow the path and investigate the fate of individual droplets in an analytical atomization source; as a result, the monodisperse droplet generator has been recognized as an exceptional tool for fundamental diagnostics in analytical atomic sources, long before the recent interest in sp-ICP work. For example, Hieftje and Malmstadt $[8,9]$ constructed a droplet generator to study various physical processes $[8,10,11]$ in an analytical chemical flame. French et al. [12] developed the "monodisperse dried microparticulate injector" (MDMI) and successfully demonstrated the injection of monodisperse droplets into an ICP. The MDMI was then adopted by a few research groups for fundamental studies in the ICP, which led to several significant discoveries on physical and chemical processes in the ICP [13-20].

The current growing interest in nanoparticle analysis with sp-ICP spectrometry creates a new demand for a better fundamental understanding of the ICP. Recent studies devoted to understanding these fundamental characteristics and behavior of the plasma when a relatively large amount of mass is introduced by a single droplet include: the local effect 
on plasma excitation conditions by an atomizing analyte droplet [21], matrix effects from injection of a single analyte droplet and particle [22], change of plasma impedance during single droplet introduction [23], and shift of radiofrequency from a free-running generator upon injection of a single droplet into the plasma [24].

Although several important fundamental ICP studies have already been performed with monodisperse droplet generators, many phenomena are yet to be clarified. For example, we have recently observed that a single microdroplet can significantly alter the plasma impedance and the coupling efficiency of radiofrequency power [23]. The objective of the present work is to understand more fully the physical behavior of the ICP during single-droplet introduction and its correlation with the change in plasma impedance

previously observed. Specifically, we monitored the size of the plasma by capturing time-resolved two-dimensional monochromatic images of the plasma, and followed its energy distribution during and after the introduction of single microdroplets. The series of captured images are compiled into a movie, which accompanies this paper as supplementary material. Representative frames that show specific effects of droplet introduction on the plasma behavior are selected and discussed in detail.

\section{Experimental}

The experimental setup consists of an ICP generator, a set of entrance optics, a monochromatic imaging spectrometer equipped an intensified charged-coupled device (ICCD) detector, a commercial monodisperse microdroplet dispenser, an in-house built trigger circuit to signal droplet introduction into the plasma, and two separate devices for measurement of any droplet-induced change in plasma impedance. 


\subsection{ICP System and Operating Conditions}

The ICP system and its operating conditions were identical to those described in our earlier singlet-droplet ICP work [23, 25]. Briefly, the ICP generator (HFP-2500F, Plasma-Therm, Kresson, NJ, USA) was crystal-controlled at 27.12 MHz. Typical outer and intermediate gas flows were used and were set at 16 and $1 \mathrm{~L} / \mathrm{min}$, respectively, whereas a much lower than common central-channel gas flow of $0.17 \mathrm{~L} / \mathrm{min}$ was used. All gas flows were regulated by individual mass-flow controllers. The inner diameter of the torch injector was $1.5 \mathrm{~mm}$, and the ICP torch was vertically mounted. The forward power of the ICP generator was $1200 \mathrm{~W}$.

\subsection{Monochromatic Imaging Spectrometer (MIS), Optical and Detector Systems}

The operating principles and characteristics of the monochromatic imaging spectrometer are different from those in conventional wavelength-dispersive mode [26, 27]. Accordingly, the optical arrangement was different from our previous studies [23, 25], in which the Czerny-Turner spectrometer was operated in the conventional one-dimensional imaging setting. In the conventional configuration, the image is focused onto the entrance slit of the spectrometer and the two axes of the two-dimensional image at the exit focal plane of the spectrometer correspond to wavelength and a one-dimensional image of the plasma (the plasma axis that coincides with the entrance-slit axis). In contrast, the monochromatic imaging spectrometer provides a two-dimensional monochromatic image of the object under study, achieved by an optical spatial Fourier transform of the plasma image and its inverse, respectively, before and after wavelength 
dispersion by the grating. Specifically, this optical Fourier transform (and its inverse) were achieved by a pair of converging lenses through collimation and refocusing [28, 29].

The monochromatic imaging spectrometer employed here is the one previously described by Sesi et al. [27], but with some modifications. The plasma observation region could be precisely selected because the plasma-torch housing was mounted onto a motorized three-dimensional translation stage. Emission from the plasma was first collimated by a fused-silica bi-convex lens with a focal length of $1.1 \mathrm{~m}$, placed one focal length away from the plasma, before arriving at the entrance slit of the spectrometer. The height and width of the entrance and exit slits were $3 \mathrm{~mm}$ and $2 \mathrm{~mm}$, respectively. The focal length of the spectrometer was $1 \mathrm{~m}$ and grating density was 2400 grooves $/ \mathrm{mm}$. Consequently, although a comparatively wide slit was used, the spectral bandpass was less than $1 \mathrm{~nm}$ (around $0.8 \mathrm{~nm}$ ) and is adequate for the emission lines employed in this work. After wavelength selection, the 2D image of the plasma was reconstructed by another fusedsilica bi-convex lens (focal length $=45 \mathrm{~cm}$ ) positioned after the exit slit of the spectrometer, and focused onto the ICCD detector. The ICCD detector employed in this study was upgraded to a newer model (iStar 734i, Andor, Ireland), with pixel dimensions of $1024 \times 1024$. To enhance signal-to-noise ratios, the pixels were binned in groups of $4 \times 4$; as a result, the dimensions of the captured images were $256 \times 256$.

The load-coil region of the plasma was the primary focus of this work. Accordingly, the observation region started about $15 \mathrm{~mm}$ below and extended to around $1 \mathrm{~mm}$ above the top of the load coil. The top turn of the load coil was located on the opposite side of the torch from the spectrometer and cast no shadow onto the ICCD image; the highest 
portion of the coil that appears in all presented images in this study corresponds to approximately $3 \mathrm{~mm}$ below the top of the load coil. Nevertheless, for ease of discussion and to match their appearance on the displayed images, the three sections of the shadow cast by the load coil onto the ICCD images are labelled as the lowest, middle and last turn of the load coil.

\subsection{Monodisperse Microdroplet Generator and Triggering System for ICCD}

The monodisperse microdroplet generator and the triggering system were identical to those previously described [23, 25]. Briefly, a commercial monodisperse microdroplet dispenser (MD-K-150-010, Microdrop GmbH, Norderstedt, Germany), capable of producing on demand a train of reproducible microdroplets of $50 \mu \mathrm{m}$ diameter (i.e., $65 \mathrm{pL}$ ) was mounted directly onto the base of the ICP torch via a glass ball-joint adaptor. Monodisperse water droplets were introduced into the plasma at a rate of $40 \mathrm{~Hz}$. The droplet stream was entrained by a flow of argon at $0.17 \mathrm{~L} / \mathrm{min}$, and travelled vertically into the base of the ICP. Droplet introduction into the ICP was signaled by atomichydrogen emission (the $\mathrm{H} \alpha$ line at $655.6 \mathrm{~nm}$ ), which was collected between the lowest and middle turns of the load coil, transmitted through a narrow-bandpass interference filter selective for the Ho emission line ( $\lambda=656.4 \mathrm{~nm}, \Delta \lambda=1 \mathrm{~nm}, 656 \mathrm{FS} 02-25$, Andover Corp., Salem, NH, USA), and measured with a PMT [23, 25]. The ac component of this photocurrent from the $\mathrm{H} \alpha$ line was amplified and used to trigger the timing circuit of the ICCD. Because solvent evaporation starts when the droplet leaves the capillary of the droplet dispenser, water vapor, which accelerates faster, reaches the plasma earlier than the droplet. As a result, the onset of hydrogen emission from the ICP is earlier, by 
$>100 \mathrm{~s} \mu \mathrm{s}$, than entrance of the intact droplet into the plasma. We have previously measured timing jitter in the Sr analyte emission signal with respect to this $\mathrm{H}$-emission trigger [25], and found that it to be less than $100 \mu$ s (typical SD $\sim 25 \mu \mathrm{s}$ ). The $\mathrm{H}$ emission suffices as a trigger for the ICCD imaging system.

\subsection{Indirect Plasma Impedance Measurement}

As outlined in our previous report [23], the plasma impedance was indirectly monitored by means of a RF-probe coil placed inside the plasma-torch compartment [30] and also through a phase-detector signal tapped directly from the impedance-matching network. The bandwidths of the RF-probe coil and the phase-detector measurement circuits were previously measured and were found to be $1,300 \mathrm{~Hz}$ and $15,000 \mathrm{~Hz}$, respectively, at $-3 \mathrm{~dB}$ attenuation [23].

Signals from the RF-probe coil, the phase detector, and the H $\alpha$-PMT were simultaneously measured by a multi-channel digital oscilloscope (TDS 2024, Tektronix, Beaverton, OR, USA). The oscilloscope traces were transferred to a computer for further processing and storage. The RF-probe coil signal was measured in ac-coupling mode whereas dc-coupling were used for other signals. To aid in identifying different stages of the impedance change, the first derivatives of the temporal profiles were also calculated. Because both the RF-probe coil and phase-detector signals were comparatively weak and noisy, and noise is worsened by differentiation, the data in the measured temporal profiles are grouped in units of 25 and then averaged. Because the digitization length of the oscilloscope is limited to 2500 data points, the time base of the oscilloscope was set 
to record only a relatively short temporal profile $(10 \mathrm{~ms})$. This arrangement provides a 100-data point temporal profile after data grouping and averaging, with sufficient signalto-noise ratio for calculation of the first derivative.

\subsection{Experimental Procedures and Data Processing}

Deionized water used throughout this study was passed through a $0.45-\mu \mathrm{m}$ nylon syringe filter before being fed into the reservoir of the droplet dispenser. To follow the changes in size and appearance of the plasma during microdroplet introduction, 100 sequential ICCD images were taken, at each studied wavelength, with increasing delay time in steps of $100 \mu \mathrm{s}$. The gate widths for all ICCD images were kept constant at $100 \mu \mathrm{s}$. In the following discussion, the time stamp on images refers to the center of the ICCD gate from the onset of the H $\alpha$-PMT trigger signal. For example, an ICCD image captured at a delay time of $500 \mu$ s with a gate width of $100 \mu$ s has a time stamp of 0.55 ms.

Three wavelengths were measured: $309.0 \mathrm{~nm}(\mathrm{OH}$ molecular band), $656.3 \mathrm{~nm}$ (H I emission) and $696.5 \mathrm{~nm}$ (Ar I emission). The gain setting for the ICCD was 40 (out of 255) for the strong Ar emission and 80 for $\mathrm{OH}$ and $\mathrm{H}$ emission. To improve the signalto-noise ratios, signals from multiple gate events were accumulated before readout. The integrated exposure time for each ICCD image was $10 \mathrm{~s}$ (corresponding to 400 droplet introductions) for the $\mathrm{OH}$ molecular band and 5 s (i.e., 200 droplets) for both Ar I and H I emission.

Plasma background emission was corrected by subtraction with an ICCD image taken with the same settings, but at a delay time of $19 \mathrm{~ms}$. Although the jitter of the H $\alpha$ signal 
to trigger the arrival time of the current droplet is less than $100 \mu$ s (with typical SD $\sim 25 \mu \mathrm{s}$ ) [25], the use of this signal to trigger the arrival time of the next droplet can be as large as $4 \mathrm{~ms}$, as measured in our previous study [25]. In other words, for the droplet dispenser operating at a nominal rate of $40 \mathrm{~Hz}$, the time difference between successive droplets reaching the plasma can range from $21 \mathrm{~ms}$ to $29 \mathrm{~ms}$. In this work, the plasma image at 19 -ms delay after the $\mathrm{H} \alpha$ trigger was chosen to faithfully represent the plasma background because any plasma perturbation brought on by the droplet introduction should have decayed away, and it is at least two milliseconds ahead of the next droplet.

\section{Results and Discussion}

\subsection{Raw OH, H and Ar Emission Images and Optical Distortion in the MIS}

Figure 1 shows the raw (i.e., before background correction) monochromatic images during single-droplet introduction into the plasma at 309, 656.3 and $696.5 \mathrm{~nm}$, which correspond to $\mathrm{OH}$ molecular, $\mathrm{H} \mathrm{I}$, and Ar I atomic emission, respectively. This set of images was taken 3.05 ms after the Ho-PMT trigger, when the droplet was located between the middle and last (upper) turns of the load coil. The presentation of raw images here serves two purposes. First, comparisons between raw and backgroundcorrected images, which will be presented in Figure 2, enable us to gauge the change in emission induced by droplet introduction relative to the plasma background. Second, because of an optical artifact in the monochromatic imaging spectrometer, the images are distorted and their vertical and horizontal scales are not identical; the dark outer regions 
in these raw images provide a convenient estimation of the true vertical and horizontal scales.

As is clear in Figure 1, the plasma appears wider with the spectrometer set at $309 \mathrm{~nm}$ than in the two images captured at 656.3 and $696.5 \mathrm{~nm}$. Distortion along the horizontal axis of the image is a typical artifact of the monochromatic imaging spectrometer. The cause has been detailed before [31], so only a brief explanation is given here. For different wavelength settings on a Czerny-Turner spectrometer, the angles of incidence (i.e., incoming) and diffraction (i.e., outgoing) beams on the grating are different; consequently, the final image becomes distorted horizontally to an extent dependent on the grating angle (i.e., wavelength setting). This effect is analogous to projecting a circular light beam perpendicularly onto a screen, which would cause the beam to appear narrower when viewed by an observer standing at an angle to the screen [31]. In the present study, the images were progressively narrowed as the spectrometer was set to longer wavelengths (i.e., when the angles of incidence and diffraction were increasingly dissimilar). Methods to correct this distortion, either through hardware (e.g., dual spectrometer arrangements [31]) or software (e.g., computer algorithms [32]), have been developed. Because the present work involves no image overlay across different wavelengths, such a rigorous correction was not applied. Nevertheless, an estimation of the extent of image distortion is informative.

The extent of image distortion induced by the monochromatic imaging spectrometer can be gauged from the true physical dimensions of features in the raw ICCD images. The ICP load coil is comprised of copper tubing of $1 / 8 ”(\sim 3.2 \mathrm{~mm})$ diameter; its shadow on 
the images provides a convenient internal marker for the vertical scales of the images. For all images, this full vertical scale represented a $16.5 \mathrm{~mm}$ height in the plasma. The horizontal scale of the image can be approximated from the plasma width. The plasma is confined within the ICP torch, which had an internal diameter of $17.8 \mathrm{~mm}$. The actual plasma width should be slightly less than the torch diameter. Therefore, to a first approximation, if the plasma fitted tightly inside the horizontal axis of the ICCD image, the image distortion, defined as the percent shrinkage of the image in the horizontal to the vertical scale, was approximately $8 \%$. The measured plasma image, at locations inside the load-coil region, covered about $83 \%$ of the full ICCD width at a wavelength of $309 \mathrm{~nm}$ and around 73\% at both $656 \mathrm{~nm}$ and $696 \mathrm{~nm}$. Therefore, the estimated image distortions were approximately $24 \%$ for the $\mathrm{OH}$ image and 33\% for both the $\mathrm{H}$ and $\mathrm{Ar}$ images reported in this paper.

Even without any background correction, both $\mathrm{OH}$ molecular and atomic $\mathrm{H}$ emission from the vaporizing droplets are strong enough to be readily recognized from the raw ICCD images (cf. Figure 1). The red dot in center of the $309 \mathrm{~nm}$ raw image corresponds dominantly to $\mathrm{OH}$ molecular emission from the vaporizing droplets, whereas the ICP continuum at $309 \mathrm{~nm}$ contributes the surrounding emission. Similarly, H $\alpha$ emission from the vaporizing droplets was strong and appears as a red dot in the image center. In contrast, there was no clear marking in the raw Ar $696.5 \mathrm{~nm}$ image; the droplet effect on the argon emission is masked by its own strong emission and is apparent only after background subtraction. It is interesting to note that the plasma emission at $309 \mathrm{~nm}$ (continuum) and of the Ar I line are skewed asymmetrically on opposite sides of the plasma. Plasma asymmetry, in particular inside this energy-addition region, is likely a 
result of the helical-shaped load coil. In addition, some distortions are found at the two sides of the plasma images. Because the load-coil region of the plasma can be viewed only through the curved plasma torch, some optical aberrations in the plasma image are likely present, especially on the two sides where the torch curvature is the steepest along the optical axis of the spectrometer.

\subsection{Local Cooling and Plasma Pinch Effects during Single Microdroplet Introduction}

Figure 2 presents the same set of ICCD images as in Figure 1, but after subtraction of plasma background emission. As the load coil is opaque and does not emit, the net emission in the load-coil region represents the noise level and provides a convenient indictor of signal-to-noise ratio (in this particular context, signal refers to the induced change in emission by the microdroplet) in these images. The net $\mathrm{OH}$ molecular emission from the vaporizing droplets becomes even clearer after background subtraction and is in the shape of an elongated bullet. In Figure 1, the previously mentioned image distortion, which was estimated to be about $24 \%$ at a wavelength of $309 \mathrm{~nm}$, contributes only minimally to the observed elongation of the $\mathrm{OH}$ emission. After correction for optical distortion, the estimated width and height of the $\mathrm{OH}$ emission cloud were about 1.1 and $1.7 \mathrm{~mm}$, respectively. The major cause of this elongated shape is upward droplet movement during the ICCD exposure, which was set at $100 \mu \mathrm{s}$. The radial distribution of $\mathrm{OH}$ emission is narrow (only $1.1 \mathrm{~mm}$ ) and confined to the immediate vicinity of the vaporizing droplet. The localized $\mathrm{OH}$ emission suggests that the decomposition kinetics of $\mathrm{OH}$ radical into $\mathrm{H}$ and $\mathrm{O}$ atoms is rapid in the ICP. This hypothesis is supported by the 
work of Olesik [14], who investigated the production kinetics of molecules, atoms and ions in the ICP by introducing yttrium-containing monodisperse droplets into the plasma, and reported that the molecular YO and atomic Y I and Y II emission all began to rise at the same time. More importantly, the YO emission was reported to last only for about $100 \mu$ s [14] indicating the fast dissociation kinetics for diatomic molecules inside the ICP.

In contrast, hydrogen atomic emission has spread throughout the whole plasma, even though the strongest $\mathrm{H}$ emission was also found in the close vicinity of the vaporizing droplet. This latter point is not surprising, since the vaporizing droplet acts as a local reservoir for the supply of $\mathrm{H}$ atoms through $\mathrm{H}_{2} \mathrm{O}$ decomposition. The dispersed $\mathrm{H}$ emission cloud agrees with the observations of Niemax and co-workers [21]; they reported that the diffusion of hydrogen atoms is much more rapid than the transport velocity of the argon plasma gas and, as a result, a significant fraction of hydrogen atoms are traveling in the backward direction inside the ICP (i.e., recirculated) and form convection currents [21]. The diffuse $\mathrm{H}$ emission can be attributed to two factors: First, the diffusion coefficient of $\mathrm{H}$ atoms is large because of its light mass; not only can hydrogen atoms diffuse rapidly throughout the plasma, they can also undergo circulation within the load-coil region [21]. Therefore, the observed $\mathrm{H}$ emission cloud includes hydrogen atoms not only from molecules vaporized and atomized from the evaporating droplet during the $100 \mu$ s ICCD exposure, but also atoms that had already atomized, diffused and even circulated, from the very beginning of the desolvation-atomization process. Second, the ionization efficiency of hydrogen is relatively low, so a comparatively large fraction of the hydrogen remains in the form of neutral atoms. 
The net change in Ar emission during single-droplet introduction is dramatically different from the behavior of $\mathrm{OH}$ and $\mathrm{H}$. At the center of the image (cf. Figure 2), the vaporizing droplet cools the plasma significantly and leads to a substantial decrease in argon emission. Because both droplet desolvation and subsequent atomization consume comparatively large amounts of energy [33], they are good candidates for causing the local cooling effect.

The cooling effect from a vaporizing droplet reduces not only the argon atomic emission, but also the plasma continuum. Surrounding the strong $\mathrm{OH}$ molecular emission (the red bullet in Figure 2), there is a sharp reduction in emission. As the net emission is negative (i.e., even lower than at steady state, which contains no $\mathrm{OH}$ emission), this emission reduction must correspond to the plasma continuum background at $309 \mathrm{~nm}$. However, this $\mathrm{OH}$ image explicitly reveals that the plasma emission is affected differently upstream and downstream from a vaporizing droplet; cooling is more significant downstream but appears negligible upstream. This asymmetrical appearance is the result of two mechanisms with opposite effects operating downstream and upstream of a vaporizing droplet, and will be discussed in detail in the next two sections. There is a slight emission enhancement upstream from the strong $\mathrm{OH}$ bullet-shaped emission cloud. This non-localized upstream enhancement is presumably also due to plasma continuum at $309 \mathrm{~nm}$, for two reasons. First, as will be discussed in later sections, this enhancement correlates well with the enhancement in Ar emission found in all studied emission images. As the plasma continuum in the ICP originates from either free-free or free-bound recombination of Ar ions, this close correlation with Ar images suggests that the slight enhancement upstream from the vaporizing droplet is due to the plasma continuum 
emission at $309 \mathrm{~nm}$. Second, the observation that this non-localized region with comparatively slight emission enhancement is found mainly upstream from a vaporizing droplet (i.e., only after the droplet passes through a particular observation volume) argues against this enhancement being a result of $\mathrm{OH}$ molecular emission. If this emission enhancement were due to $\mathrm{OH}$ molecular emission, the $\mathrm{OH}$ radicals would be expected to spread to regions both upstream and downstream from the vaporizing droplet, through similar mechanisms as in the case of hydrogen emission. Therefore, it is most likely that the non-localized changes in the $\mathrm{OH}$ emission images are due to the plasma continuum at $309 \mathrm{~nm}$ rather than $\mathrm{OH}$ molecular emission.

Apart from local cooling, the droplet also causes a sharp drop in Ar $696.5 \mathrm{~nm}$ emission along the two sides of the plasma. In other words, the radial dimension of the plasma is reduced. This shrinkage is the result of a thermal pinch effect caused by the droplet. Thermal pinches in the ICP are common when a foreign substance (e.g., organic solvent [34, 35] or molecular gas [36-39]), which has a higher thermal conductivity than the plasma gas (i.e., argon), is introduced into the plasma. In brief, the higher thermal conductivity accelerates heat conduction (and hence heat loss) from the plasma; the effect is particularly acute across the steep thermal gradients at its boundary, which then cools the peripheral regions of the plasma [34]. When the outer surface of the plasma cools, its electrical conductivity also drops and the plasma volume shrinks [34, 38]; consequently, the power density in the plasma goes up. In the present experiment, both phenomena (thermal pinch - plasma shrinking and more energetic plasma) were observed. They will be discussed in more detail in Section 3.4. 
The effect of an aqueous aerosol and its desolvation on the analytical performance and fundamental characteristics of an ICP has been widely reported in the literature [37, 4046]. Overall, an aqueous aerosol induces a complex inter-relationship between solvent loading and the plasma operating conditions [43, 44]. A heavy burden of introduced aerosol (e.g., by means of a conventional nebulizer-spray chamber setup, which typically sends more than $10^{6}$ droplets per second into the plasma $\left.[15,47]\right)$ normally cools the plasma and degrades its analytical performance, whereas a light loading of an aerosol (in particular in the form of water vapor) improves energy transfer within the plasma and results in a more energetic discharge [37, 45, 46]. Hydrogen, released from the dissociation of water molecules, has a thermal conductivity over one order of magnitude higher than that of argon [36, 45], and is regarded as the likely primary cause for the enhanced energy transfer in the ICP $[43,45,48]$. Similarly, because a plasma pinch originates from a change in plasma thermal conductivity, the plasma pinch effect observed during single microdroplet introduction is also probably attributable to hydrogen generated from the dissociation of water.

\subsection{Volume of Local Plasma Cooling Caused by a Vaporizing Droplet}

Figure 3 shows the net emission images for $\mathrm{OH} 309$ nm, H I $656.3 \mathrm{~nm}$ and Ar I $696.5 \mathrm{~nm}$ at $1.15 \mathrm{~ms}$ after the Ho-PMT trigger. The vaporizing droplet appears just above the lowest turn of the load coil. This set of images was chosen because it clearly shows the cooled volume of the plasma at a relatively early stage during droplet introduction. Figure 3 contrasts with Figure 2 in two important ways. First, the cooled volume is much larger in Fig. 3. The estimated widths, after correction for optical distortion, that exhibit 
a clear decline in plasma continuum at $309 \mathrm{~nm}$ and in Ar I $696.5 \mathrm{~nm}$ emission are about $5.9 \mathrm{~mm}$ and $6.0 \mathrm{~mm}$, respectively, at $1.15 \mathrm{~ms}$ (cf. Figure 3), whereas the same widths are only $3.6 \mathrm{~mm}$ and $3.4 \mathrm{~mm}$, respectively, at $3.05 \mathrm{~ms}$ (cf. Figure 2). The substantial difference in the widths of the cooled volume is likely related to the droplet size, which is expected to be significantly larger at $1.15 \mathrm{~ms}$ than at $3.05 \mathrm{~ms}$. A brief glance at Figures 2 and 3 might lead one to conclude that the cooling effect on Ar is greater at $3.05 \mathrm{~ms}$ because of the larger net reduction in Ar emission and apparent darker cooled region. However, it is necessary to consider also the steady-state Ar intensity distribution (cf. Figure 1), which is significantly higher between the middle and last turns of the load coil. As will be discussed in the next section, the percentage decrease in Ar emission is very similar in both cases.

The second distinction between Figures 2 and 3 is that the $\mathrm{OH}$ emission cloud at $1.15 \mathrm{~ms}$ (Figure 3) appears much broader than that at $3.05 \mathrm{~ms}$ (Figure 2); this difference is probably attributable to differences in the local plasma temperature. It seems likely that the plasma temperature in the vicinity of the droplet is higher in the upper regions of the plasma (i.e., at a longer delay time), which would promote the rapid dissociation of $\mathrm{OH}$ and result in a smaller zone of strong $\mathrm{OH}$ emission. In particular, the significantly wider region cooled by the larger droplet (cf. Figure 3) implies that heat transfer to the droplet is less efficient and the volume around the droplet is made cooler. Another possibility is that the injector gas has a strong impact on the plasma temperature, in particular in the region where the entering cool argon gas interacts with the hot plasma (i.e., the central channel in the lower part of the load-coil region). Computer simulation [22] has shown that, with an injector-gas flow of $0.14 \mathrm{~L} / \mathrm{min}$, the central channel from the injector exit up 
to the bottom of the lowest turn of the load coil is cooled by the incoming argon. Moreover, with a modest increase in argon flow to $0.25 \mathrm{~L} / \mathrm{min}$, the cooling extends to midway between the lowest and the middle turns of the load coil [22]. Therefore, it is probable that the plasma temperature, even in the absence of droplet introduction, is lower around the bottom-most turn of the load coil. Consequently, a lower plasma temperature would allow the $\mathrm{OH}$ radicals to travel farther away from the droplet (hence wider emission cloud), before significant molecular $\mathrm{OH}$ dissociation occurs.

The $\mathrm{OH}$ and Ar background-corrected emission images (cf. Figures 2 and 3) also reveal that plasma cooling extends not only radially from the droplet, but also downstream (upward) in the plasma. In both cases, the cooling spreads to around $6 \mathrm{~mm}$ downstream from the vaporizing droplet. This cooled zone is much larger than would be expected based on results previously reported. Olesik and Fister [14, 49] studied the local cooling from vaporizing droplets and reported that the cooling can reach $2 \mathrm{~mm}$ from the physical location of a droplet. It is difficult to directly compare our current results with those of Olesik and Fister [14, 49] because of the very different experimental conditions. First, Olesik and Fister [49] employed a conventional concentric nebulizer coupled with a Scott-type spray chamber. The spray chamber is very effective in removing droplets larger than about $25 \mu \mathrm{m}$, which was experimentally confirmed with measurements at the exit of the spray chamber $[49,50]$. In addition, Olesik and Fister [49] performed the experiment at much higher observation positions (all were above the load coil), which means that the largest incompletely vaporized droplets are below the $25 \mu \mathrm{m}$ limit. The much more extended cooling volume observed in the present experiment is likely related to the much larger droplet size in the plasma. 
Another interesting feature in Figure 3 is the thermal pinch effect. Even while the droplet is still low in the plasma, plasma shrinkage (i.e., the decrease in Ar emission at the plasma boundary) already extends at least to the plasma region between the middle and the last (top) turns of the load coil (i.e., almost the entire load-coil region). Also, hydrogen has already spread throughout the load-coil region at this early time. This correlation of hydrogen diffusion and the plasma pinch effect further supports the hypothesis that hydrogen plays an important role in causing the plasma pinch. The accompanying movie (in electronic supplementary information) illustrates the progressive change in plasma net emission (i.e., after subtraction of steady-state plasma background continuum) at these three monitored wavelengths. In these images, the hydrogen emission cloud is always dispersed and begins to spread into the plasma as early as $0.15 \mathrm{~ms}$ - almost $1 \mathrm{~ms}$ before the appearance of $\mathrm{OH}$ emission. At $0.65 \mathrm{~ms}$, when the droplet is still within the lowest turn of the load coil and therefore is occluded, hydrogen emission covers most of the load-coil region and a clear plasma-pinch effect exists between the middle and the top turns of the load coil (see Section 3.6 for further discussion).

\subsection{Plasma Reheating Upstream of a Vaporizing Droplet}

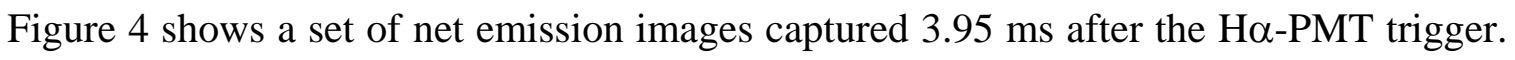
This is the second to the last set of images in which the vaporizing droplet was still within the imaging area of the MIS, and just slightly below the top of the load coil located on the side opposite to the spectrometer. The droplet moved completely out of the imaging area at $4.15 \mathrm{~ms}$. The most striking feature in this set of images is reheating 
of the plasma after the passage of the vaporizing droplet. Upstream from (below) the vaporizing droplet, the net emission at $309 \mathrm{~nm}$, which in this case corresponds to the plasma continuum at $309 \mathrm{~nm}$, and emission at Ar I $696.5 \mathrm{~nm}$ are both positive. The positive net emission indicates that both the plasma continuum and Ar emission have not only fully recovered from the cooling effect induced by a passing droplet, but have grown even stronger than at steady state. In other words, the plasma becomes hotter once the vaporizing droplet passes through a particular volume.

Figure 5 shows the percentage change in Ar I 696.5 nm emission at three different times (1.15, 3.05 and $3.95 \mathrm{~ms}$ ) after the H $\alpha$-PMT trigger, times that correspond to the images displayed in Figures 2, 1 and 3, respectively. For all figures presented in this paper, percentage change in emission refers to the net change (i.e., after subtraction of steadystate emission) relative to the steady-state emission. Optical thickness of the Ar line was not determined in the present experiment. A published collisional-radiative model [51] indicated that there should be some significant self absorption in $\mathrm{Ar} 4 \mathrm{p}-4 \mathrm{~s}$ radiative transitions, which includes the Ar I $696.5 \mathrm{~nm}$ line employed in the present study. Nevertheless, the percentage change in Ar emission presented in Figure 5 and other figures in the present study should offer a qualitative and probably a semi-quantitative indication of the effect that a microdroplet has on Ar emission. Unlike the cooling effect, which is localized, the reheating effect is diffuse and relatively even throughout the discharge. In addition, the reheating is a fast process (relative to the time scale studied in this experiment, which was $100 \mu \mathrm{s})$. Once the droplet passes through a particular observation volume, that volume is already reheated in the next ICCD frame. Figures 4 
and 5 clearly depict that the reheating zone closely follows the local-cooling zone and that the boundary between these two opposite effects is only $\sim 1 \mathrm{~mm}$ thick.

From the foregoing discussion, a vaporizing droplet first causes a strong local cooling, but the portion of the plasma that was cooled is almost immediately reheated to an even higher temperature than the steady state once the droplet moves away. Figure 5 shows that the maximum percentage drop in Ar emission, due to local cooling by the vaporizing droplet, is very similar at different times and was about $-10 \%$ in all three cases. Such an observation is rather unexpected as the droplet size is certainly shrinking during its passage in the ICP. The extent of plasma reheating is, of course, time-dependent. The maximum overshoot of Ar emission was about 5\%, as shown in the case at $3.95 \mathrm{~ms}$ presented in Figure 5 and at other studied times. Niemax and co-workers [21] also observed local cooling followed by plasma reheating during single microdroplet injection. With a spectrometric system consisting of three monochromators and PMTs, Groh et al. [21] monitored the time-dependent ICP emission of $\mathrm{H} \mathrm{I}$, Si I and Ar I lines from a nanoparticle of silicon dioxide embedded in a 52- $\mu \mathrm{m}$ microdroplet, and reported that the Ar I emission dropped 4\% due to plasma cooling by the droplet but then increased and overshot by about $1 \%$ the value before droplet injection. Of course, the change in $\mathrm{Ar}$ emission observed by Groh et al. [21] is a spatially averaged value, and could be significantly different from the spatially resolved line-of-sight changes measured in the current study. 


\subsection{Aftereffect of Microdroplet Introduction into Plasma}

After the microdroplet moves out of the load-coil region, both the plasma continuum at $309 \mathrm{~nm}$ and Ar emission start to shift back to equilibrium. However, it takes a comparatively long time for the reheating effect to decay. Figure 6 shows the set of background-corrected emission images at the three studied wavelengths at $6.15 \mathrm{~ms}$, which is $2.0 \mathrm{~ms}$ after the droplet has moved out of the observation zone. By this time the $\mathrm{H}$ emission has decayed considerably and become faint; its emission between the middle and the last (top) turns of the load coil was only 13\% of that at $3.95 \mathrm{~ms}$ and only $3 \%$ compared to its maximum at $3.05 \mathrm{~ms}$. As will be shown in greater detail in the next section, the plasma pinch effect has also decayed substantially and has nearly vanished. Yet, plasma reheating is still apparent, as reflected by the well defined net increase in both plasma continuum and Ar emission.

Because of noise levels in the images, it is experimentally difficult to determine the exact time when the plasma-reheating effect subsides and Ar emission returns to its steady-state level. A semi-quantitative method is therefore used here, based on color change in the majority of pixels in the image representing percentage change of Ar emission. Figure 7 shows the percentage change (i.e., net change in emission relative to the steady state) in

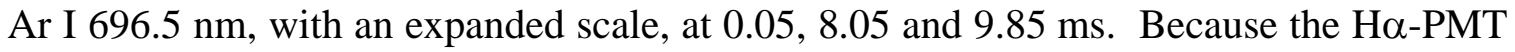
trigger fires before the microdroplet enters the plasma, the percentage change in $\mathrm{Ar}$ emission at $0.05 \mathrm{~ms}$ should reflect plasma conditions that are practically identical to the steady state. In other words, no change in Ar emission is expected at $0.05 \mathrm{~ms}$; any observed percentage change in Ar emission should represent the noise level of the 
measurement, and thus establish a comparison baseline for other times. Most pixels in this baseline-reference image (at $0.05 \mathrm{~ms}$ ) display colors between light blue and light green, indicating that the reproducibility of Ar-emission measurement was about $\pm 1.5 \%$. When images captured at other times are compared to this reference, the presence of plasma reheating is deemed to exist only when the candidate image shows substantially more pixels that are yellow, orange or red (i.e., percentage change of Ar emission greater than the $1.5 \%$ noise threshold) than in the reference.

The percentage change in the Ar emission image at 8.05 ms clearly displays substantially more pixels in yellow and orange than the reference at $0.05 \mathrm{~ms}$. Therefore, the plasma at 8.05 ms has not yet returned to steady-state equilibrium and, although weak, plasma reheating is still underway. In contrast, the difference between the image captured at $9.85 \mathrm{~ms}$ and the reference at $0.05 \mathrm{~ms}$ is marginal; therefore, it is difficult to judge with confidence whether plasma reheating continues at $9.85 \mathrm{~ms}$. Nevertheless, the positive confirmation of a plasma-reheating effect at $8.05 \mathrm{~ms}$, about $4 \mathrm{~ms}$ after the microdroplet left the load-coil region of the plasma, indicates that restoration of plasma equilibrium after the perturbation induced by microdroplet injection is slow.

\subsection{Correlation with Change in Plasma Impedance}

In our previous paper [23], we reported that a momentary change in plasma impedance occurs upon single microdroplet introduction. Temporal spikes, lasting about $10 \mathrm{~ms}$, in both the RF-probe coil and the phase-detector signals were observed during droplet introduction [23]. Even when the automatic impedance-matching network was disabled, 
the same temporal spikes in both signals were found, implying that the long-lasting disturbance of plasma impedance is not due to an artifact caused by the automatic impedance-tuning circuitry [23]. To clarify the cause of this impedance change, the position of the microdroplet inside the plasma, the plasma pinch and the plasma reheating effects were all correlated with the temporal change in plasma impedance.

Along with capture of the ICP monochromatic images presented above, the temporal behavior of the signals from the H $\alpha$ PMT, the RF-probe coil and the phase detector were simultaneously measured during repetitive single-droplet introduction, and are presented in Figure 8a. The error bars represent the standard deviations from four measurements, each measured at a different wavelength setting of the monochromatic imaging spectrometer. To ensure that there was no significant drift in plasma conditions during the course of the study, monochromatic images at a chosen wavelength were captured twice, one at the start and one at the end of the experiment. All three traces in Figure 8a approached, but did not decay back to, the steady-state level at $8.8 \mathrm{~ms}$. In Figure 8a, the H $\alpha$-PMT signal represents not only $\mathrm{H}$ I emission but also the plasma background continuum (i.e., no background correction was applied); therefore, it is unclear if the slow

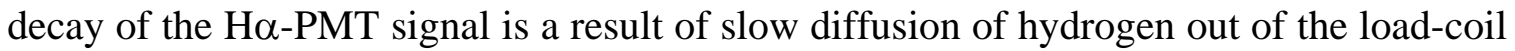
region, a brighter plasma continuum due to plasma reheating, or a combination of both. Because the temporal features are broad, it is difficult to assign them to a particular cause. To better pinpoint the behavioral transitions and correlate them with the monochromatic images, first derivatives of the temporal profiles were calculated. 
Figure 8b shows the rate of change (first derivative) of the RF-probe and phase-detector signals as a function of time. Because these two derivatives have opposite polarities, the phase-detector signal was inverted to better match the directional change of the RF-probe coil output. Overall, the first derivatives of the two signals reflecting plasma-impedance changes correlate well; yet the RF-probe coil lagged behind the phase-detector signal at most times. For example, the maximum rate of change for the phase-detector signal occurred at $\sim 0.9 \mathrm{~ms}$ (marked by arrow I) whereas that from the RF-probe appeared $\sim 0.5 \mathrm{~ms}$ later, at $\sim 1.4 \mathrm{~ms}$. Similarly, the maximum signals (i.e., zero derivatives) for the phase-detector and RF-probe signals were at $\sim 2.8$ and $3.1 \mathrm{~ms}$, respectively. These relatively small differences could possibly be artifacts caused by the different temporal responses of the two measurement circuits. In our previous work, the bandwidths, at $-3 \mathrm{~dB}$ attenuation, of the RF-probe coil and phase-detector circuits were experimentally determined to be 1,300 and 15,000 Hz, respectively [23]. The slight lag of the RF-probe coil response falls within the uncertainty dictated by this bandwidth. Because of the wider bandwidth of the phase-detector circuit, its temporal response will be used as a reference to correlate the plasma-impedance change with droplet position and plasma appearance. Four important transitions in plasma-impedance change have been identified and are marked with arrows in Figures 8a and 8b.

The first important transition occurred at $\sim 0.9$ ms (marked arrow I) when the phasedetector derivative attained its peak (i.e., highest rate of impedance change). This corresponded to the moment when the thermal pinch and plasma shrinkage became established in the load-coil region of the plasma. Although the H $\alpha$-PMT signal was also at its maximum at this time, the correlation between the maximum derivative in phase- 
detector signal and the H $\alpha$-PMT signal could be just a coincidence; at this particular time,

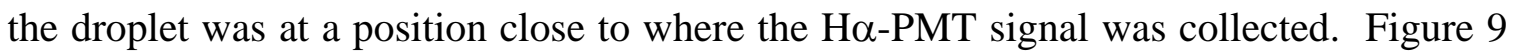
shows the percentage change in Ar emission, relative to steady state, at $0.45,0.75$ and 1.05 ms. Plasma shrinkage starts to develop at $0.45 \mathrm{~ms}$; a tiny reduction in Ar emission can be observed on both sides of the plasma between the lowest and the middle turns of the load coil. A further drop in Ar emission occurs in the lower load-coil region and plasma shrinkage progresses to the last (top) turn of the load coil at $0.75 \mathrm{~ms}$. At $1.05 \mathrm{~ms}$, the thermal pinch effect is apparent both within and above the load-coil region of the plasma.

The second transition was found at $\sim 2.8$ ms (marked arrow II in Figure 8b) when the phase-detector derivative transitions from positive to negative (i.e., the temporal peak in plasma impedance change, cf. Figure 8a), and corresponds to the time when the microdroplet was close to the center of the load-coil region. The emission images are omitted here as they were very similar to those presented for $3.05 \mathrm{~ms}$ in Figure 2. The third transition arose at $\sim 4.2 \mathrm{~ms}$ (marked arrow III in Figure 8b) when the rate of change in phase-detector signal attained its maximum in the direction opposite that of the first transition, and before it began to return to the baseline level (cf. Figures 8a and 8b). This moment was very close to the time when the droplet moved out of the load-coil region, which was determined to be $\sim 4.05 \mathrm{~ms}$ in the present study. The plasma images at the third transition are similar to those presented for $3.95 \mathrm{~ms}$ in Figure 4. Recall that there is another half turn of load coil on the opposite side of the torch from the spectrometer; the droplet in Figure 4 was therefore still within the load-coil region. 
The final transition occurred at $~ 5.8 \mathrm{~ms}$ (marked arrow IV in Figure $8 \mathrm{~b}$ ), in which the rate of change in phase-detector signal began to roll off as a horizontal line. It was found that plasma shrinkage started to ease around this time. The three images in Figure 10 show the percentage change in Ar I $696.5 \mathrm{~nm}$ emission, relative to steady state, at 5.15, 5.75 and $6.35 \mathrm{~ms}$. At $5.15 \mathrm{~ms}$, the plasma shrinkage is still apparent. At $5.75 \mathrm{~ms}$, the reduction in Ar emission is less but yet noticeable. At $6.35 \mathrm{~ms}$, the reduction in Ar emission on the two sides of the plasma further diminishes; compared to the image captured at $5.15 \mathrm{~ms}$, most of the plasma shrinkage has subsided.

Hydrogen liberated from dissociation of water molecules modifies the thermal conductivity of the plasma, induces a plasma pinch effect and causes the plasma to shrink. Naturally, as the plasma narrows and its thermal conductivity goes up, plasma impedance as well as radiofrequency coupling efficiency also vary. This hypothesis is supported by the correlation among the different stages of changes in plasma impedance, the droplet positions and the thermal pinch effects during single microdroplet introduction. However, the reason that a relatively long time is needed before the plasma impedance returns to its steady-state level is not clear. One possible and simple reason is that the change in plasma impedance is very sensitive and non-linear to the presence of hydrogen and/or to the plasma pinch effect in the plasma. Under this hypothesis, even though these effects have largely decayed, a trace amount of hydrogen remaining in the plasma might still be sufficient to induce a measurable change in plasma impedance, and it probably takes an additional few milliseconds to completely flush all the remaining hydrogen out of the plasma. 
Another potential reason for the long decay time for plasma impedance is related to the finite time required for heat conduction and energy transfer to occur. Although the present study provides no evidence for this hypothesis, it is worth considering this possibility as a guide for design of future experiments. It is known that radiofrequency coupling into the plasma is not uniform and that the strongest coupling occurs within the skin depth of the plasma [52, 53]. Rodham et al. [54], by means of power interruption, studied the radial transport of energy from the periphery to the center the plasma within the load coil region, and concluded that energy coupling and thus heating initially occurs in the outer region of the plasma and that it takes about $1 \mathrm{~ms}$ to move into the center of the plasma [54]. Moreover, the electrical conductivity of argon is a strong function of temperature [55]; as a result, with other factors identical, power is more effectively coupled into the hot zone than into the cooler region [54]. Rodham et al. [54] also observed the development of hot spots inside the load-coil region of the ICP during their power-interruption experiment. As shown in the present study, the plasma is reheated to an even higher temperature after the passage of a droplet, which then provides positive feedback to further change the energy coupling. To restore the energy-coupling equilibrium, not only must hydrogen be completely removed from the system but also the temperature distribution among plasma species needs to be re-established. However, temperature redistribution in an atmospheric-pressure argon plasma by thermal diffusion and ambipolar diffusion is slow [56]. For an ICP with an 18-mm diameter and an assumed temperature of $10,000 \mathrm{~K}$, the time constants, which are the time required for the system to approach 1/e (i.e., 37\%) of the asymptotic values, for thermal conduction [57] and ambipolar diffusion [58] are calculated to be $1.0 \mathrm{~ms}$ and $5.4 \mathrm{~ms}$, respectively. 
Therefore, it is reasonable to expect that, even after complete flushing of hydrogen from the plasma, it would still take a few milliseconds to completely restore the temperature equilibrium of the plasma.

\section{Conclusion}

The introduction of a single micrometer-sized droplet causes a significant perturbation in an ICP. By means of monochromatic imaging, we observed plasma shrinkage due to a thermal pinch, significant cooling of the plasma downstream from a vaporizing droplet, and plasma reheating upstream from the droplet. Hydrogen, which has a much higher thermal conductivity than argon, likely plays an important role in the thermal pinch and reheating effects, whereas the vaporizing droplet acts as a local heat sink and cools the

plasma. For a $50-\mu \mathrm{m}$ diameter droplet, this local cooling effect is strong and extends $6 \mathrm{~mm}$ from the physical location of the droplet. The plasma-reheating effect lasts longer than $4 \mathrm{~ms}$ even after the droplet has left a particular observation volume of the plasma.

In addition to a change in size and temperature distribution within the plasma, droplet introduction causes a significant change in plasma impedance that correlates qualitatively with the different stages of plasma perturbation. It takes roughly $10 \mathrm{~ms}$ for both an RFprobe voltage and a phase-detector signal to return to the baseline level. In other words, if the microdroplet introduction rate is higher than $100 \mathrm{~Hz}$, the induced perturbation in plasma impendence is unlikely to return to a steady-state level before arrival of the next droplet. Further investigations are needed to understand this effect on the characteristics and performance of the plasma with microdroplet introduction at a high repetition rate. 


\section{Supplementary Material}

A movie, assembled from 100 sequential monochromatic images, showing the net change in emission at 309, 656.3 and $696.5 \mathrm{~nm}$ during and after introduction of single microdroplets into the ICP, is available for download from the journal website.

\section{Acknowledgements}

This research was supported by the US Department of Energy through Grant DE-FG0298ER14890 awarded to Indiana University. 


\section{References}

1. F. Laborda, E. Bolea, J. Jimenez-Lamana, Single particle inductively coupled plasma mass spectrometry: A powerful tool for nanoanalysis, Anal. Chem. 86 (2014) 22702278.

2. C.C. Garcia, A. Murtazin, S. Groh, V. Horvatic, K. Niemax, Characterization of single $\mathrm{Au}$ and $\mathrm{SiO}_{2}$ nano- and microparticles by ICP-OES using monodisperse droplets of standard solutions for calibration, J. Anal. At. Spectrom. 25 (2010) 645653.

3. O. Borovinskaya, S. Gschwind, B. Hattendorf, M. Tanner, D. Günther, Simultaneous mass quantification of nanoparticles of different composition in a mixture by microdroplet generator-icptofms, Anal. Chem. 86 (2014) 8142-8148.

4. K. Shigeta, G. Koellensperger, E. Rampler, H. Traub, L. Rottmann, U. Panne, A. Okino, N. Jakubowski, Sample introduction of single selenized yeast cells (Saccharomyces cerevisiae) by micro droplet generation into an ICP-sector field mass spectrometer for label-free detection of trace elements, J. Anal. At. Spectrom. 28 (2013) 637-645.

5. Y. Ishihara, M. Aida, A. Nomura, H. Miyahara, A. Hokura, A. Okino, Development of desolvation system for single-cell analysis using droplet injection inductively coupled plasma atomic emission spectroscopy, Anal. Sci. 31 (2015) 781-785.

6. B. Franze, I. Strenge, C. Engelhard, Single particle inductively coupled plasma mass spectrometry: Evaluation of three different pneumatic and piezo-based sample introduction systems for the characterization of silver nanoparticles, J. Anal. At. Spectrom. 27 (2012) 1074-1083.

7. S. Gschwind, M.D.L. Aja Montes, D. Günther, Comparison of sp-ICP-MS and MDG-ICP-MS for the determination of particle number concentration, Anal. Bioanal. Chem. 407 (2015) 4035-4044.

8. G.M. Hieftje, H.V. Malmstadt, A unique system for studying flame spectrometric processes, Anal. Chem. 40 (1968) 1860-1867.

9. G.M. Hieftje, H.V. Malmstadt, A new approach to flame spectrometric analysis utilizing isolated droplets of sample solution, Anal. Chem. 41 (1969) 1735-1744. 
10. N.C. Clampitt, G.M. Hieftje, Investigation into mechanism of desolvation of sample droplets in flame spectrometry, Anal. Chem. 44 (1972) 1211-1219.

11. G.J. Bastiaans, G.M. Hieftje, Investigation into vaporization of individual solute particles in flame spectrometry, Anal. Chem. 46 (1974) 901-910.

12. J.B. French, B. Etkin, R. Jong, Monodisperse dried microparticulate injector for analytical instrumentation, Anal. Chem. 66 (1994) 685-691.

13. J.W. Olesik, S.E. Hobbs, Monodisperse dried microparticulate injector - a new tool for studying fundamental processes in inductively coupled plasmas, Anal. Chem. 66 (1994) 3371-3378.

14. J.W. Olesik, Investigating fate of individual sample droplets in inductively coupled plasmas, Appl. Spectrosc. 51 (1997) A158-A175.

15. J.W. Olesik, J.A. Kinzer, G.J. McGowan, Observation of atom and ion clouds produced from single droplets of sample in inductively coupled plasmas by optical emission and laser induced fluorescence imaging, Appl. Spectrosc. 51 (1997) 607616.

16. I.I. Stewart, C.E. Hensman, J.W. Olesik, Influence of gas sampling on analyte transport within the ICP and ion sampling for ICP-MS studied using individual, isolated sample droplets, Appl. Spectrosc. 54 (2000) 164-174.

17. J.W. Olesik, J.A. Kinzer, Measurement of monodisperse droplet desolvation in an inductively coupled plasma using droplet size dependent peaks in Mie scattering intensity, Spectrochim. Acta Part B 61 (2006) 696-704.

18. L.A. Allen, J.J. Leach, R.S. Houk, Spatial location of the space charge effect in individual ion clouds using monodisperse dried microparticulate injection with a twin quadrupole inductively coupled plasma mass spectrometer, Anal. Chem. 69 (1997) 2384-2391.

19. A.C. Lazar, P.B. Farnsworth, Matrix effect studies in inductively coupled plasma with monodisperse droplets. Part I: Influence of matrix on vertical analyte emission profile, Appl. Spectrosc. 53 (1999) 457-464.

20. A.C. Lazar, P.B. Farnsworth, Matrix effect studies in inductively coupled plasma with monodisperse droplets. Part II: Influence of matrix on spatially integrated ion density, Appl. Spectrosc. 53 (1999) 465-470. 
21. S. Groh, C.C. Garcia, A. Murtazin, V. Horvatic, K. Niemax, Local effects of atomizing analyte droplets on the plasma parameters of the inductively coupled plasma, Spectrochim. Acta Part B 64 (2009) 247-254.

22. A. Murtazin, S. Groh, K. Niemax, Investigation of sample introduction- and plasmarelated matrix effects in inductively coupled plasma spectrometry applying single analyte droplet and particle injection, Spectrochim. Acta Part B 67 (2012) 3-16.

23. G.C.Y. Chan, Z.L. Zhu, G.M. Hieftje, Effect of single aerosol droplets on plasma impedance in the inductively coupled plasma, Spectrochim. Acta Part B 76 (2012) 87-95.

24. H. Miyahara, Y. Kaburaki, T. Iwai, A. Okino, Effects of droplet introduction into the ICP sustained by free-running RF-generator on plasma spectroscopic characteristics, Bunseki Kagaku 63 (2014) 109-117.

25. G.C.Y. Chan, Z.L. Zhu, G.M. Hieftje, Operating parameters and observation modes for individual droplet analysis by inductively coupled plasma-atomic emission spectrometry, Spectrochim. Acta Part B 76 (2012) 77-86.

26. J.W. Olesik, G.M. Hieftje, Optical imaging spectrometers, Anal. Chem. 57 (1985) 2049-2055.

27. N.N. Sesi, D.S. Hanselman, P.J. Galley, J.A. Horner, M. Huang, G.M. Hieftje, An imaging based instrument for fundamental plasma studies, Spectrochim. Acta Part B 52 (1997) 83-102.

28. R.A. Phillips, Spatial filtering experiments for undergraduate laboratories, Am. J. Phys. 37 (1969) 536-540.

29. C.G. Wynne, Simple Fourier-transform lenses - II, Opt. Comm. 12 (1974) 270-274.

30. G.C.Y. Chan, G.M. Hieftje, Warning indicators for the presence of plasma-related matrix effects in inductively coupled plasma-atomic emission spectrometry, J. Anal. At. Spectrom. 23 (2008) 181-192.

31. M.R. Webb, G.M. Hieftje, Improved monochromatic imaging spectrometer, Appl. Spectrosc. 60 (2006) 57-60.

32. C. Engelhard, S.J. Ray, W. Buscher, V. Hoffmann, G.M. Hieftje, Correcting distortion in a monochromatic imaging spectrometer for application to elemental 
imaging by glow discharge-optical emission spectrometry, J. Anal. At. Spectrom. 25 (2010) 1874-1881.

33. L.C. Bates, J.W. Olesik, Effect of sample aerosol transport rate on inductively coupled plasma atomic emission and fluorescence, J. Anal. At. Spectrom. 5 (1990) 239-247.

34. D.G. Weir, M.W. Blades, Characteristics of an inductively coupled argon plasma operating with organic aerosols. Part 1. Spectral and spatial observations, J. Anal. At. Spectrom. 9 (1994) 1311-1322.

35. D.G.J. Weir, M.W. Blades, The response of the inductively coupled argon plasma to solvent plasma load: Spatially resolved maps of electron density obtained from the intensity of one argon line, Spectrochim. Acta Part B 49 (1994) 1231-1250.

36. L. Ebdon, P. Goodall, Slurry atomization using hydrogen-modified inductively coupled plasmas, J. Anal. At. Spectrom. 7 (1992) 1111-1116.

37. M. Murillo, J.M. Mermet, Improvement of the energy-transfer with added-hydrogen in inductively coupled plasma atomic emission-spectrometry, Spectrochim. Acta Part B 44 (1989) 359-366.

38. E.H. Choot, G. Horlick, Vertical spatial emission profiles in $\mathrm{Ar}-\mathrm{N}_{2}$ mixed gas inductively coupled plasmas, Spectrochim. Acta Part B 41 (1986) 889-906.

39. N.N. Sesi, A. Mackenzie, K.E. Shanks, P.Y. Yang, G.M. Hieftje, Fundamental studies of mixed gas inductively coupled plasmas, Spectrochim. Acta Part B 49 (1994) 1259-1282.

40. O.T. Akinbo, J.W. Carnahan, Investigation of a flat sheet membrane desolvator for aqueous solvent removal with inductively coupled plasma atomic emission spectrometry, Talanta 45 (1997) 137-146.

41. M. Grotti, C. Lagomarsino, R. Frache, A new nebulization device with exchangeable aerosol generation mode as a useful tool to investigate sample introduction processes in inductively coupled plasma atomic emission spectrometry, Spectrochim. Acta Part B 59 (2004) 1001-1006.

42. L. Darbha, M.V. Suryanarayana, S. Natarajan, Studies on the improvement of sensitivity by heating the spray chamber in ICP-OES, Spectrochim. Acta Part B 47 (1992) 581-584. 
43. I. Novotny, J.C. Farinas, J.L. Wan, E. Poussel, J.M. Mermet, Effect of power and carrier gas flow rate on tolerance to water loading in inductively coupled plasma atomic emission spectrometry, Spectrochim. Acta Part B 51 (1996) 1517-1526.

44. G.C.Y. Chan, W.T. Chan, X.L. Mao, R.E. Russo, Comparison of matrix effects in inductively coupled plasma using laser ablation and solution nebulization for dry and wet plasma conditions, Spectrochim. Acta Part B 56 (2001) 1375-1386.

45. Y.Q. Tang, C. Trassy, Inductively coupled plasma: The role of water in axial excitation temperatures, Spectrochim. Acta Part B 41 (1986) 143-150.

46. S.E. Long, R.F. Browner, Influence of water on conditions in the inductively coupled argon plasma, Spectrochim. Acta Part B 43 (1988) 1461-1471.

47. J.W. Olesik, Fundamental research in ICP-OES and ICP-MS, Anal. Chem. 68 (1996) 469A-474A.

48. P.E. Walters, C.A. Barnardt, The role of desolvation and hydrogen addition on the excitation features of the inductively coupled plasma, Spectrochim. Acta Part B 43 (1988) 325-337.

49. J.W. Olesik, J.C. Fister, Incompletely desolvated droplets in argon inductively coupled plasmas: Their number, original size and effect on emission intensities, Spectrochim. Acta Part B 46 (1991) 851-868.

50. J.W. Olesik, L.C. Bates, Characterization of aerosols produced by pneumatic nebulizers for inductively-coupled plasma sample introduction - effect of liquid and gas flow rates on volume based drop size distributions, Spectrochim. Acta Part B 50 (1995) 285-303.

51. T. Hasegawa, H. Haraguchi, A collisional-radiative model including radiation trapping and transport phenomena for diagnostics of an inductively coupled argon plasma, Spectrochim. Acta Part B 40 (1985) 1505-1515.

52. P.B. Farnsworth, D.A. Rodham, D.W. Ririe, The use of time-resolved and spaceresolved emission data for fundamental-studies of A pulsed ICP, Spectrochim. Acta Part B 42 (1987) 393-406.

53. R.H. Scott, V.A. Fassel, R.N. Kniseley, D.E. Nixon, Inductively coupled plasmaoptical emission analytical spectrometry, Anal. Chem. 46 (1974) 75-80. 
54. D.A. Rodham, J.K. Shurtleff, P.B. Farnsworth, Energy-transport in the inductively coupled plasma, Mikrochim. Acta 3 (1989) 187-195.

55. R.S. Devoto, Transport coefficients of partially ionized argon, Physics of Fluids 10 (1967) 354-364.

56. H. Kafrouni, Study of electron cooling, diffusion and recombination in a decaying argon arc, Physica B+C 98 (1979) 100-112.

57. T. Sakuta, S. Oguri, T. Takashima, M.I. Boulos, Effects of plasma diameter and operating frequency on dynamic behaviour of induction thermal plasma, Plasma Sources Science and Technology 2 (1993) 67.

58. Y. Celik, M. Aramaki, D. Luggenhölscher, U. Czarnetzki, Determination of electron densities by diode-laser absorption spectroscopy in a pulsed ICP, Plasma Sources Science and Technology 20 (2011) 015022. 


\section{Figure Captions}

Figure 1 Raw monochromatic images (i.e., before background correction) of the ICP load-coil region at 309, 656.3 and $696.5 \mathrm{~nm}$ (as marked), during repetitive introduction of monodisperse microdroplets into the ICP. The full vertical scales in the images are the same for all three wavelengths, and represent a 16.5-mm height in the plasma. The horizontal scales in the images are wavelength-dependent; the full scales correspond to a plasma width of $21.7 \mathrm{~mm}$ for the 309-nm image and widths of $24.6 \mathrm{~mm}$ for the 656.3- and 696.5-nm images (see text for details). Time stamp $=$ $3.05 \mathrm{~ms}$.

Figure 2 Net monochromatic images (i.e., after background correction) of the ICP load-coil region at 309, 656.3 and $696.5 \mathrm{~nm}$ (as marked), during repetitive introduction of monodisperse microdroplets into the ICP. Time stamp $=$ $3.05 \mathrm{~ms}$.

Figure 3 Net monochromatic images (i.e., after background correction) of the ICP load-coil region at 309, 656.3 and $696.5 \mathrm{~nm}$ (as marked), during repetitive introduction of monodisperse microdroplets into the ICP. Time stamp = $1.15 \mathrm{~ms}$.

Figure 4 Net monochromatic images (i.e., after background correction) of the ICP load-coil region at 309, 656.3 and $696.5 \mathrm{~nm}$ (as marked), during repetitive introduction of monodisperse microdroplets into the ICP. Refer to Figure 1 for vertical and horizontal scales of the images. Time stamp = $3.95 \mathrm{~ms}$. 
Figure 5 Images showing percentage change in Ar I $696.5 \mathrm{~nm}$ emission, relative to steady state, at $1.15 \mathrm{~ms}, 3.05 \mathrm{~ms}$, and $3.95 \mathrm{~ms}$ (as marked), during repetitive introduction of monodisperse microdroplets into the ICP.

Figure 6 Net monochromatic images (i.e., after background correction) of the ICP load-coil region at 309, 656.3 and $696.5 \mathrm{~nm}$ (as marked), during repetitive introduction of monodisperse microdroplets into the ICP. Time stamp $=$ $6.15 \mathrm{~ms}$.

Figure 7 Images showing percentage change in Ar I $696.5 \mathrm{~nm}$ emission, relative to steady state, at $0.05 \mathrm{~ms}, 8.05 \mathrm{~ms}$, and $9.85 \mathrm{~ms}$ (as marked), during repetitive introduction of monodisperse microdroplets into the ICP.

Figure 8 H $\alpha$-PMT, RF-probe coil and phase-detector signals during repetitive single-droplet introduction into the ICP: (a) averaged temporal profiles, and (b) their first derivatives. The error bars represent the standard deviations from four repetitive measurements. Four notable transitions in the temporal profiles and their derivatives are marked as Arrows I, II, III and IV (see text for discussion).

Figure 9 Images showing percentage change in Ar I $696.5 \mathrm{~nm}$ emission, relative to steady state, at $0.45 \mathrm{~ms}, 0.75 \mathrm{~ms}$, and $1.05 \mathrm{~ms}$ (as marked), during repetitive introduction of monodisperse microdroplets into the ICP.

Figure 10 Images showing percentage change in Ar I $696.5 \mathrm{~nm}$ emission, relative to steady state, at $5.15 \mathrm{~ms}$, $5.75 \mathrm{~ms}$, and $6.35 \mathrm{~ms}$ (as marked), during repetitive introduction of monodisperse microdroplets into the ICP. 
Figure 1

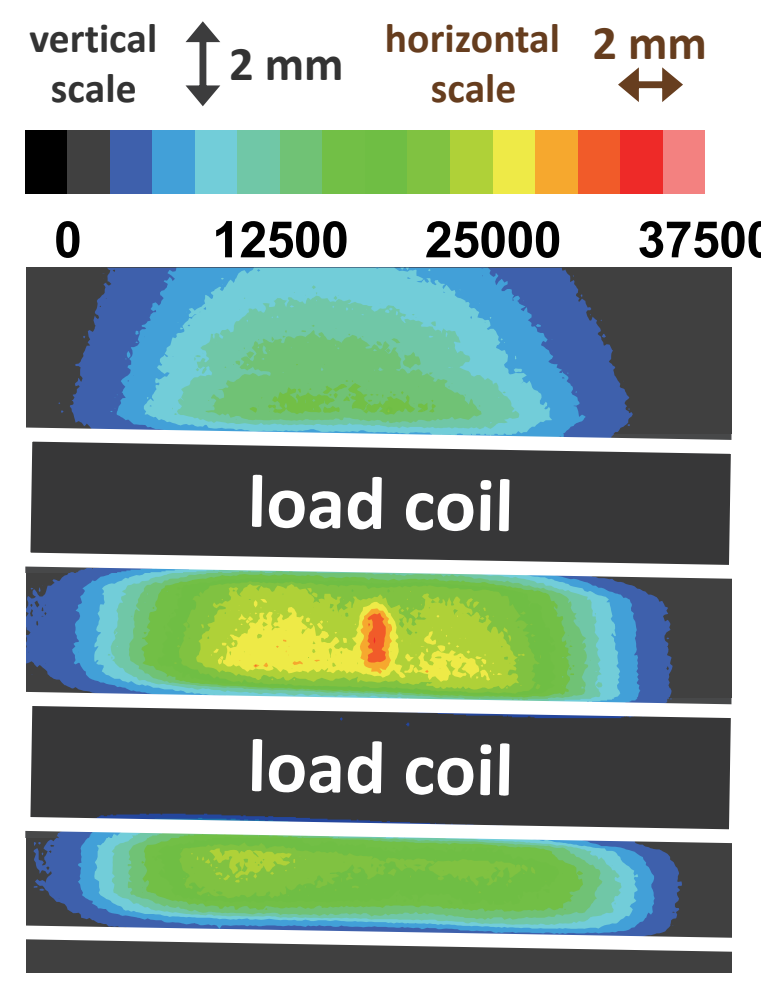

OH 309 nm
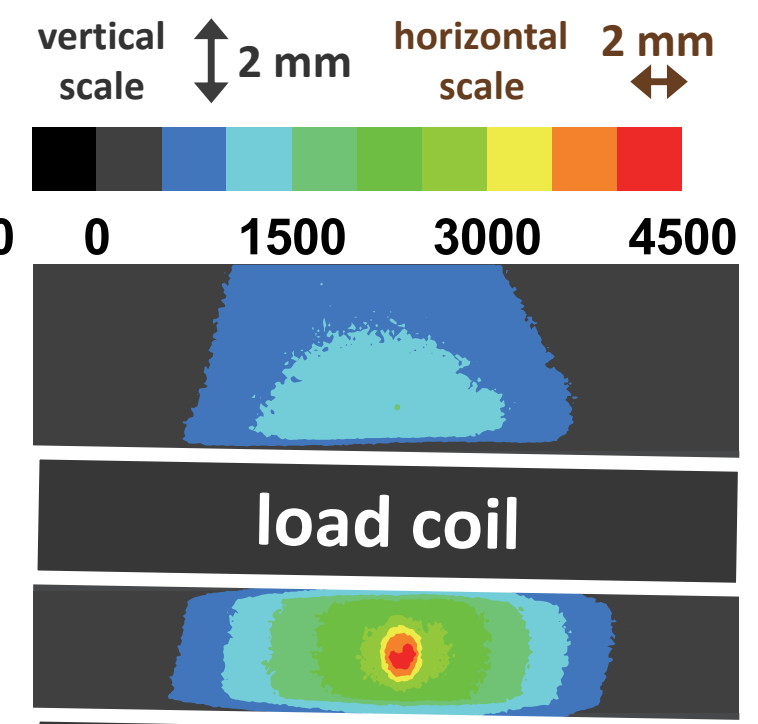

load coil

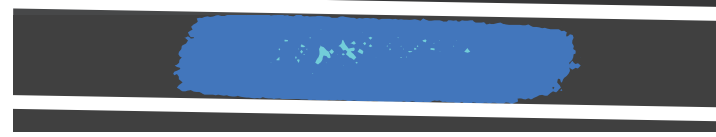

$\mathrm{H}_{\alpha} 656.3 \mathrm{~nm}$

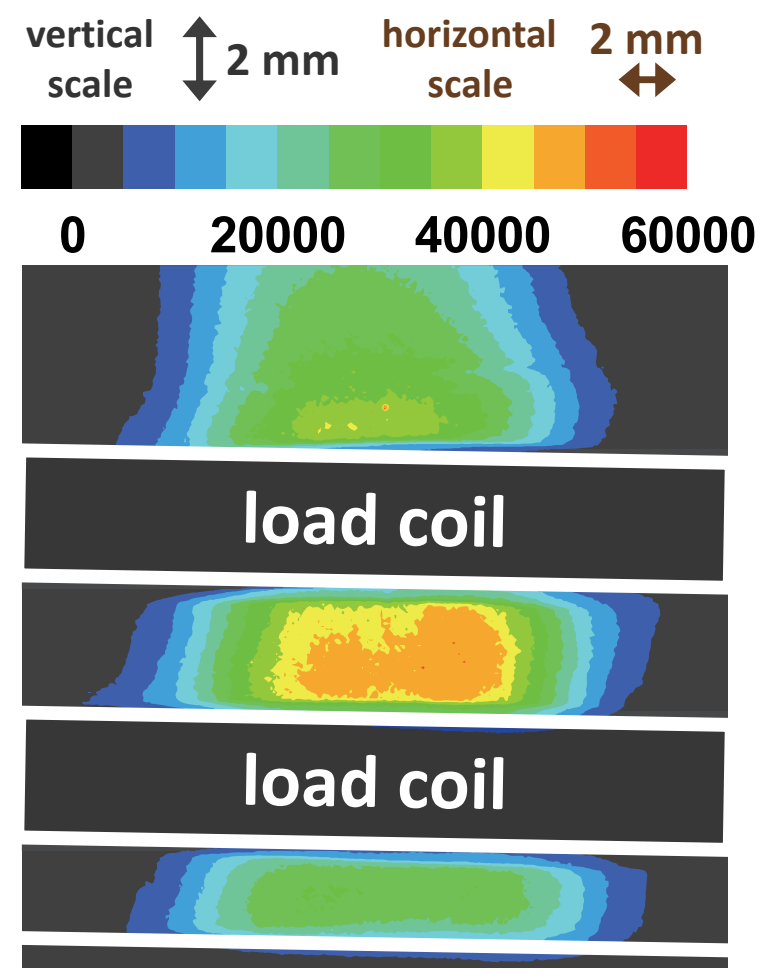

Ar 696.5 nm 
Figure 2

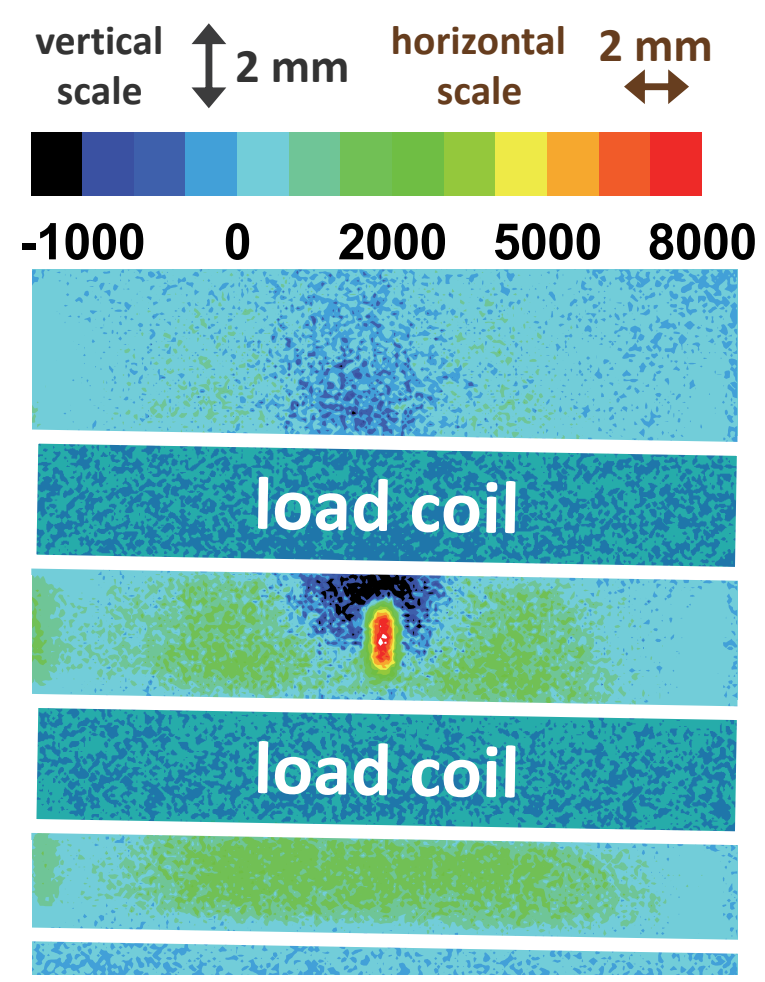

OH 309 nm

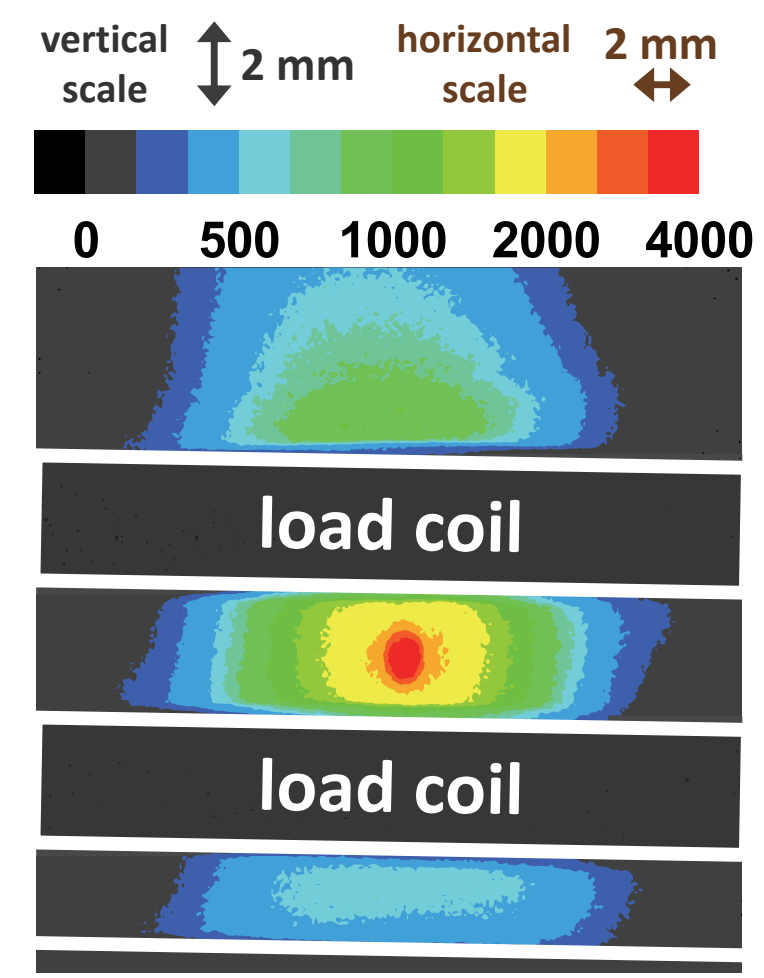

$\mathrm{H} \alpha 656.3 \mathrm{~nm}$

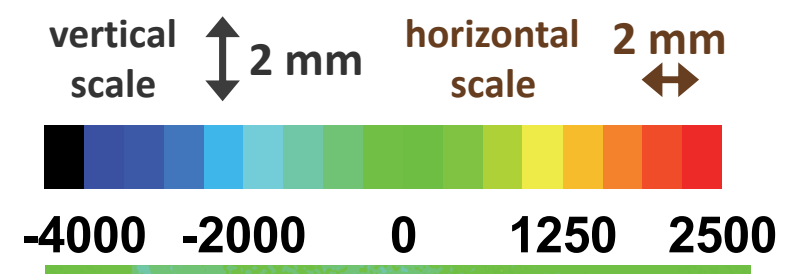

load coil

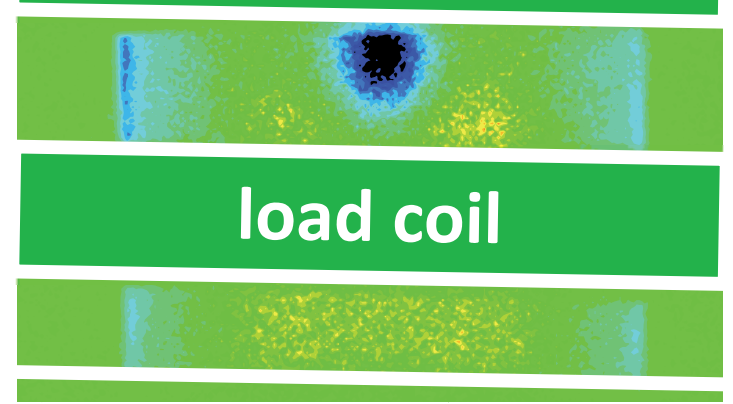

Ar 696.5 nm 
Figure 3

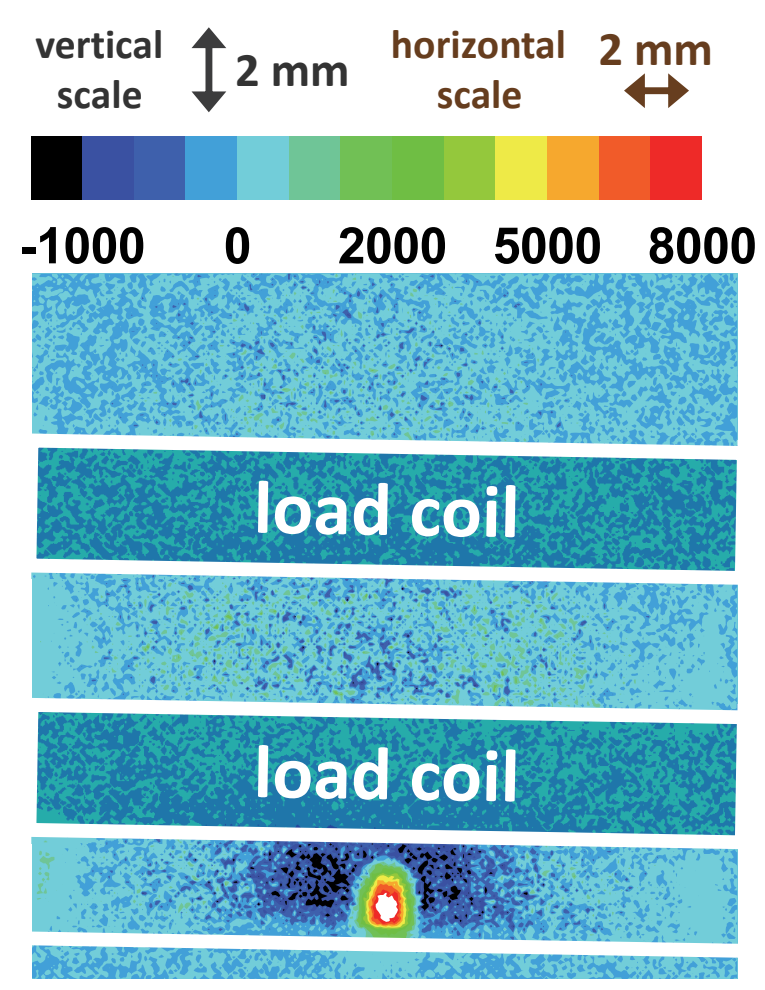

OH 309 nm

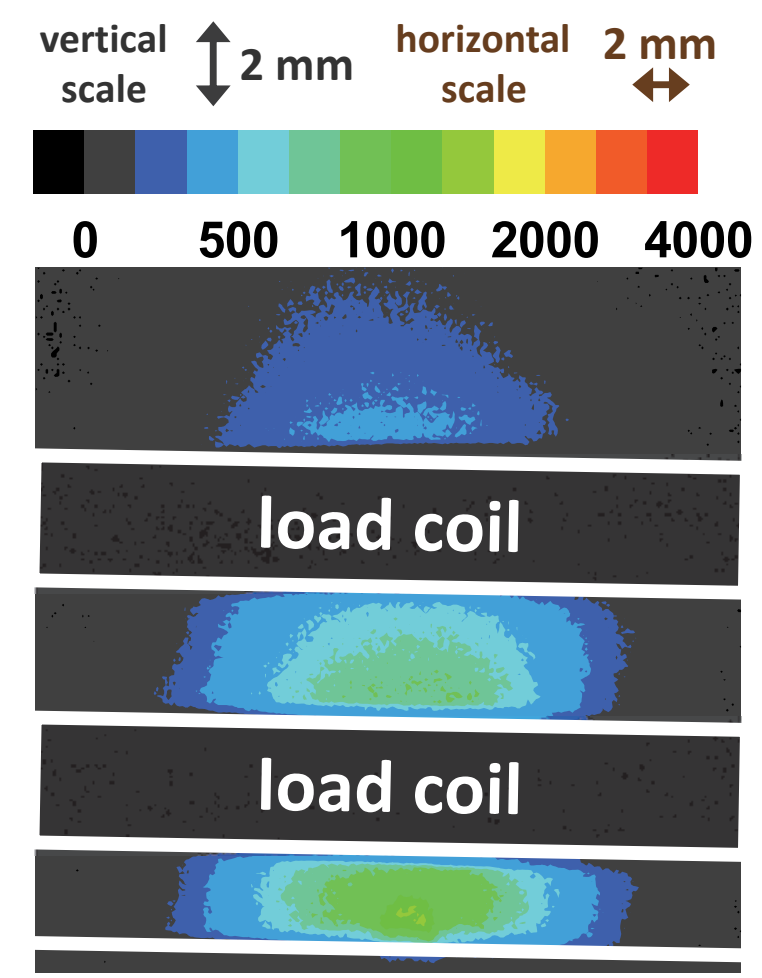

$\mathrm{H} \alpha 656.3 \mathrm{~nm}$

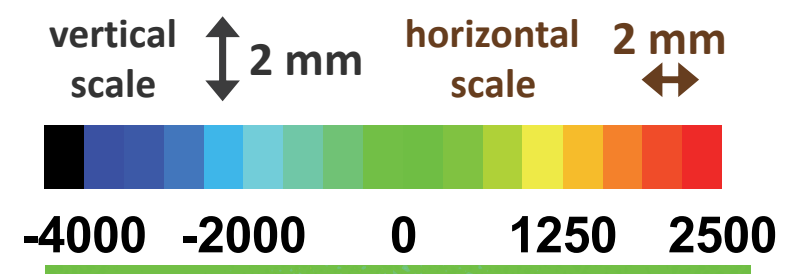

load coil

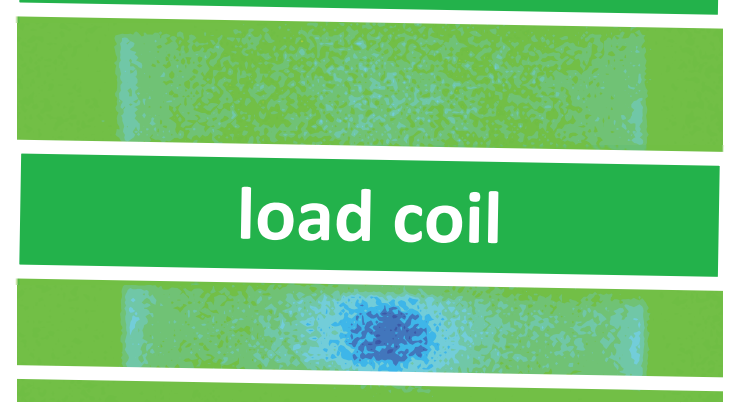

Ar 696.5 nm 
Figure 4

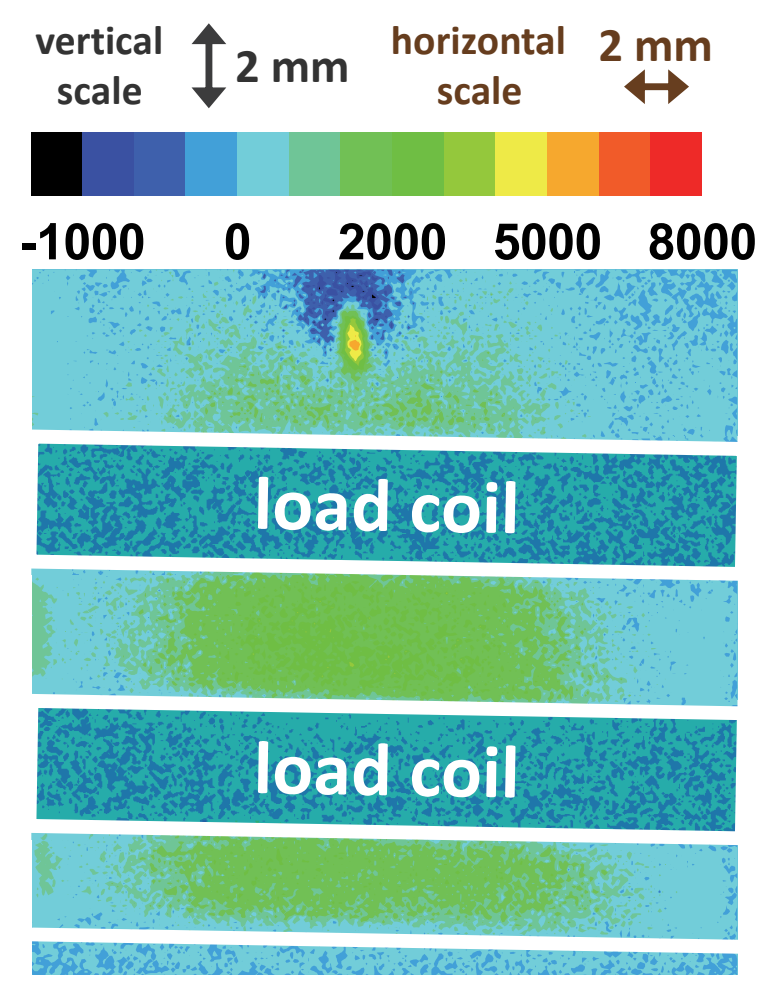

OH 309 nm

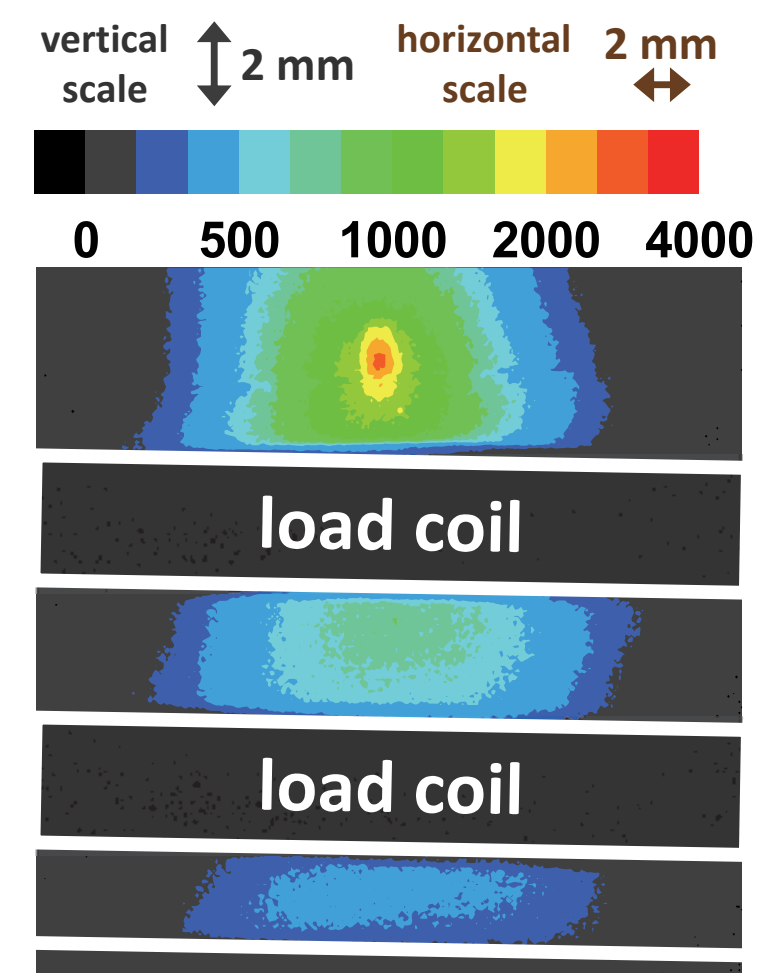

$\mathrm{H}_{\alpha} 656.3 \mathrm{~nm}$

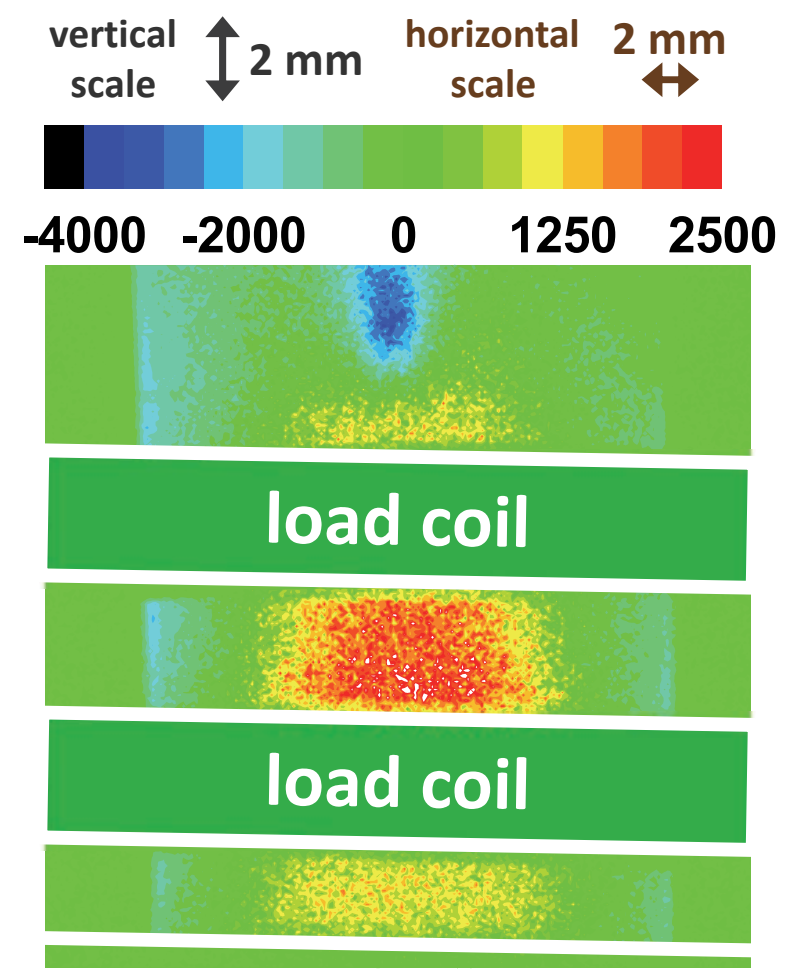

Ar 696.5 nm 
Figure 5

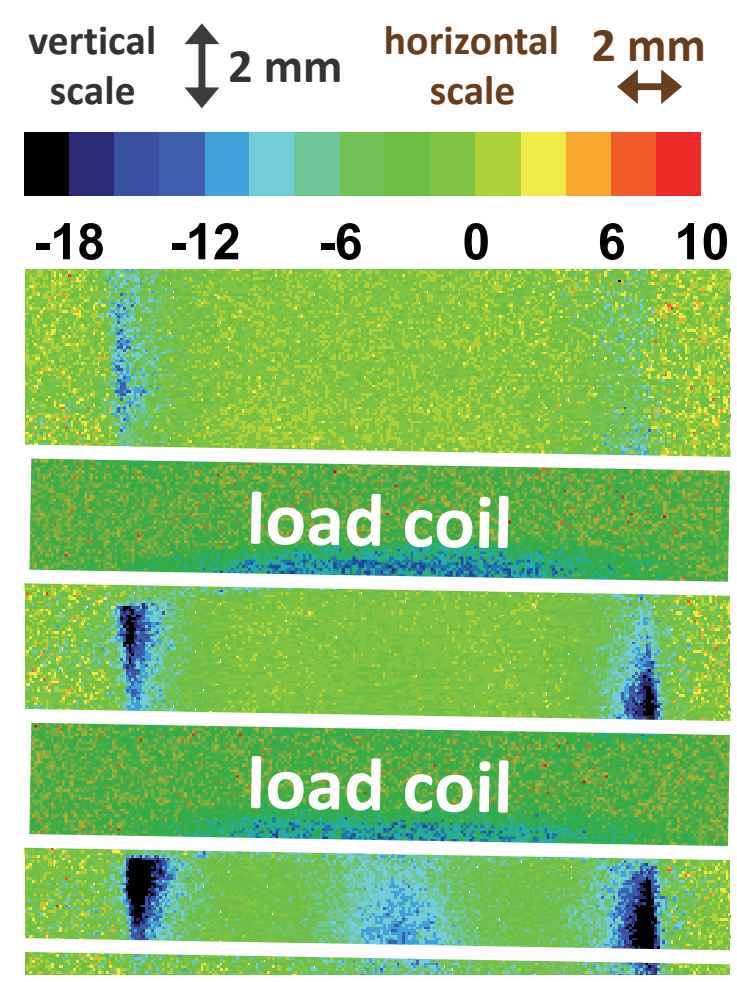

$1.15 \mathrm{~ms}$
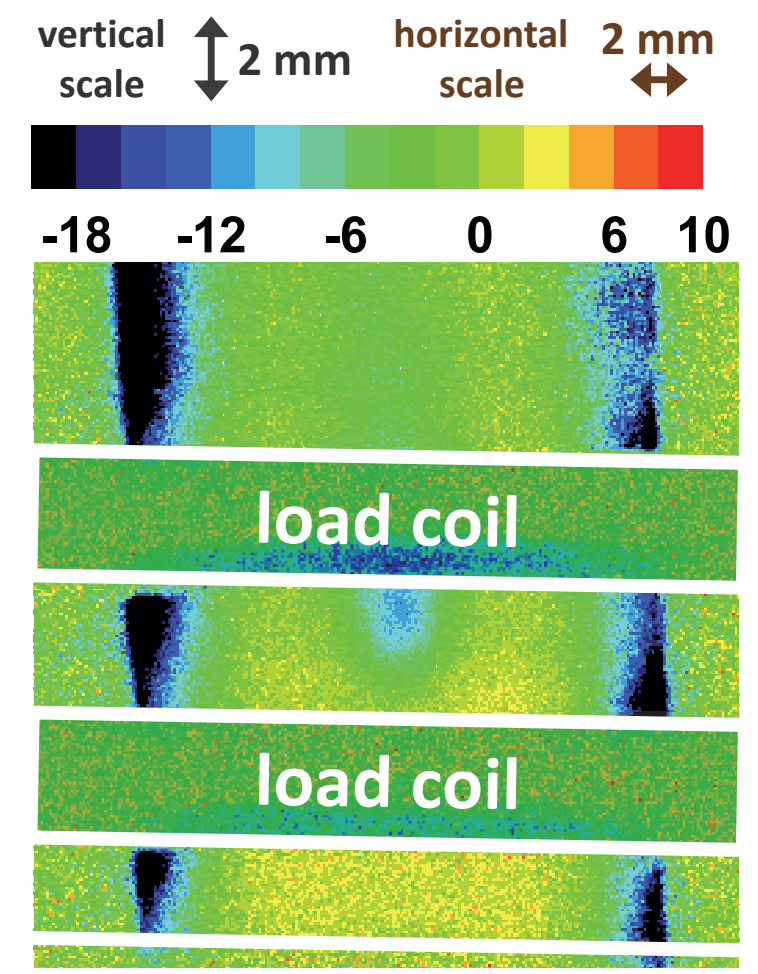

$3.05 \mathrm{~ms}$

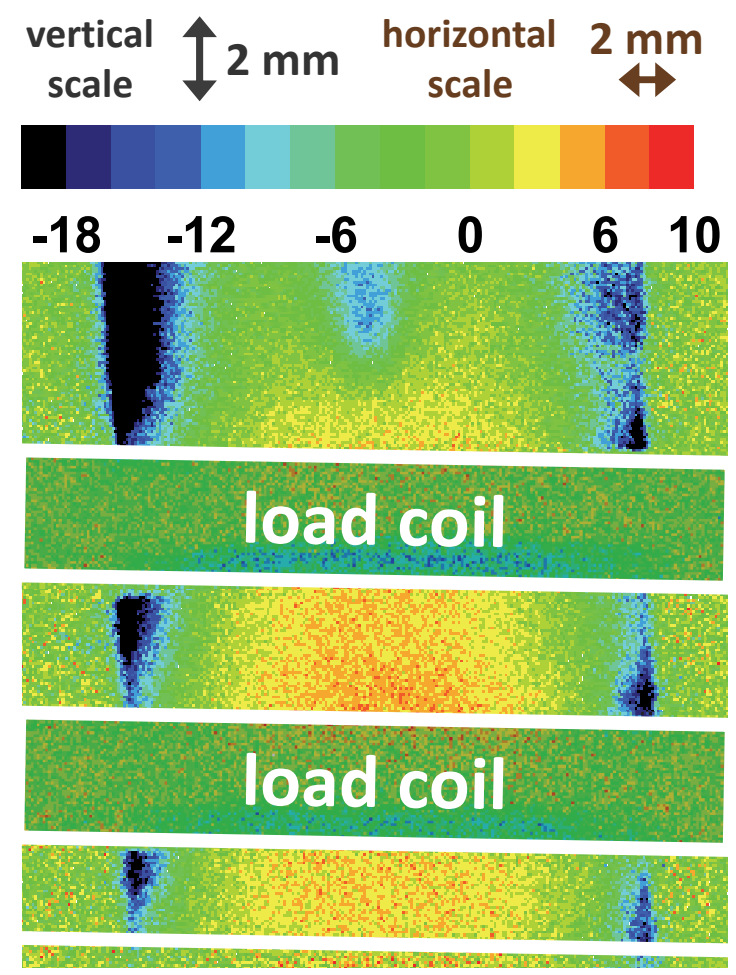

$3.95 \mathrm{~ms}$ 
Figure 6

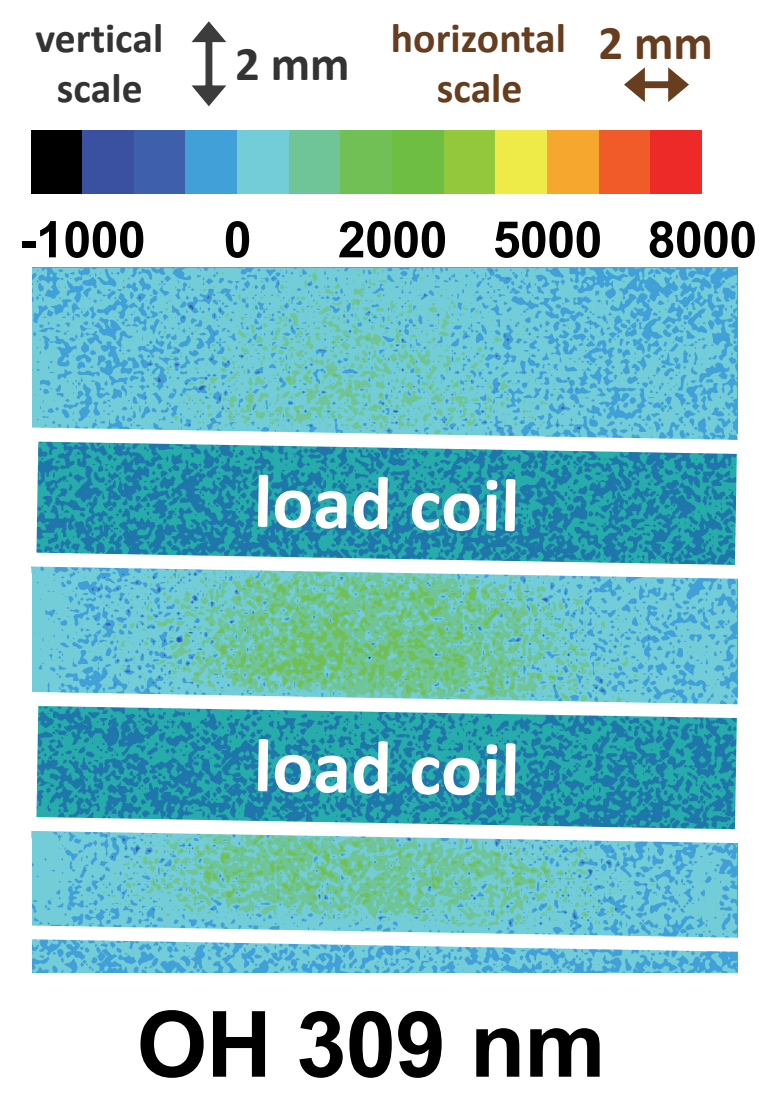

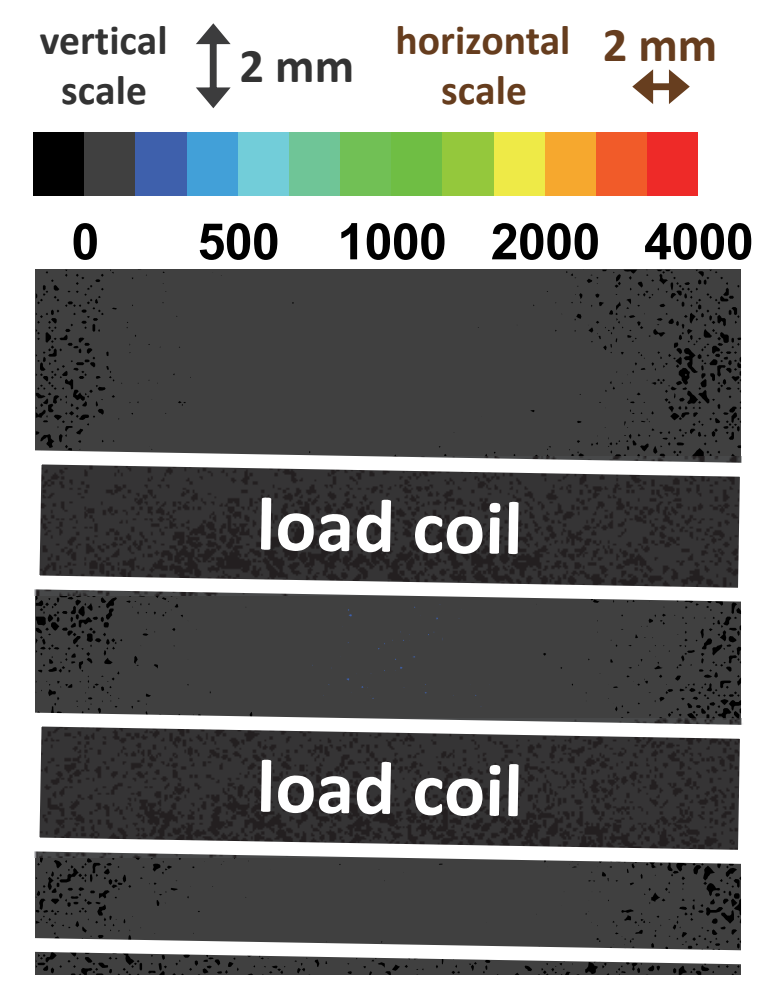

$\mathrm{H} \alpha 656.3 \mathrm{~nm}$
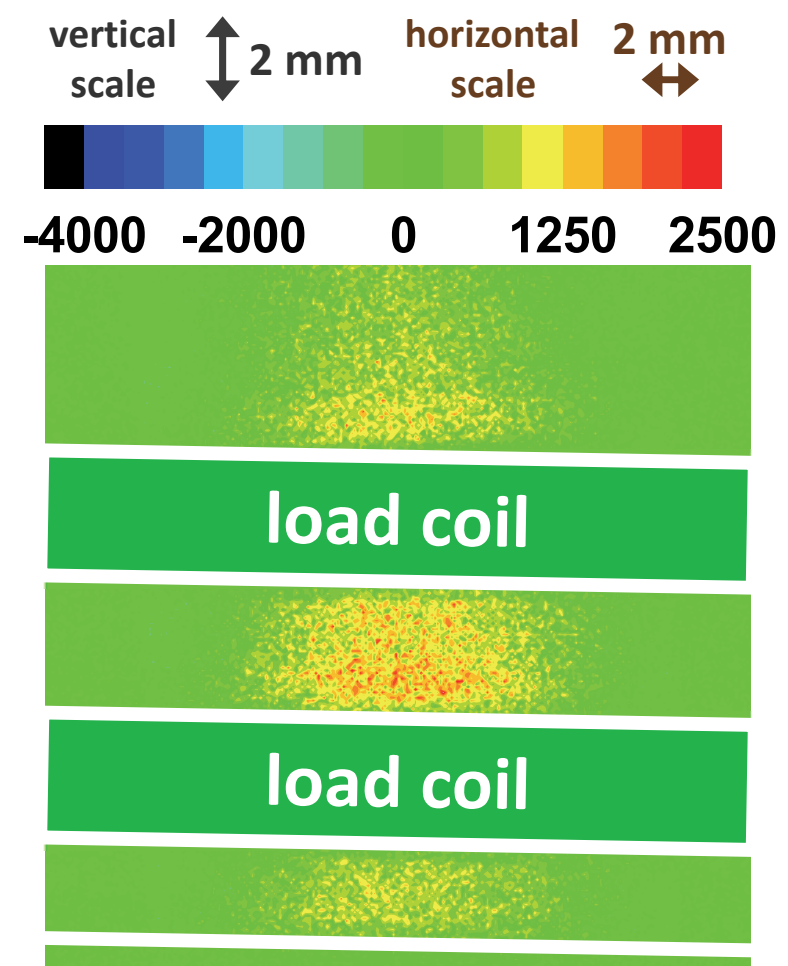

Ar 696.5 nm 
Figure 7

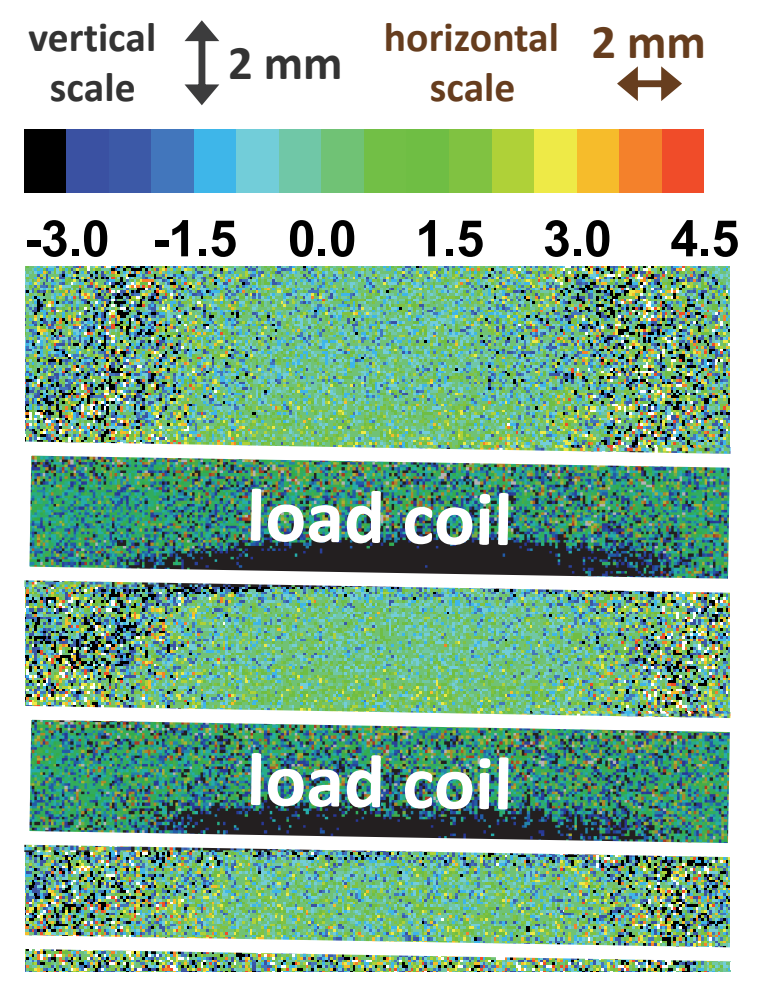

$0.05 \mathrm{~ms}$
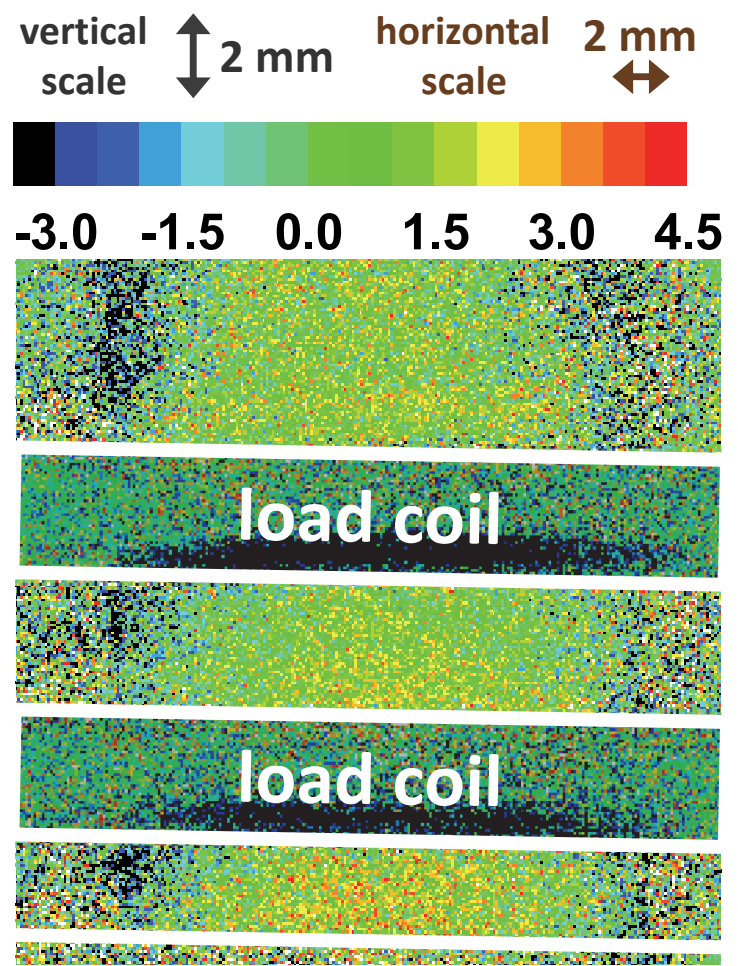

$8.05 \mathrm{~ms}$

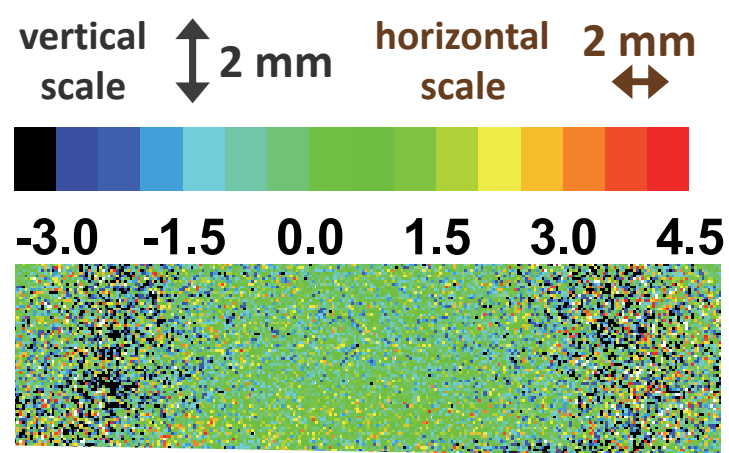

load eoll

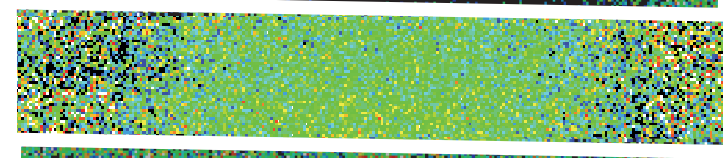

\section{load coll}

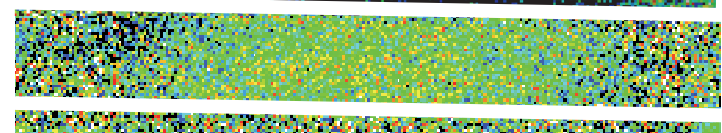

$9.85 \mathrm{~ms}$ 
Figure 8a

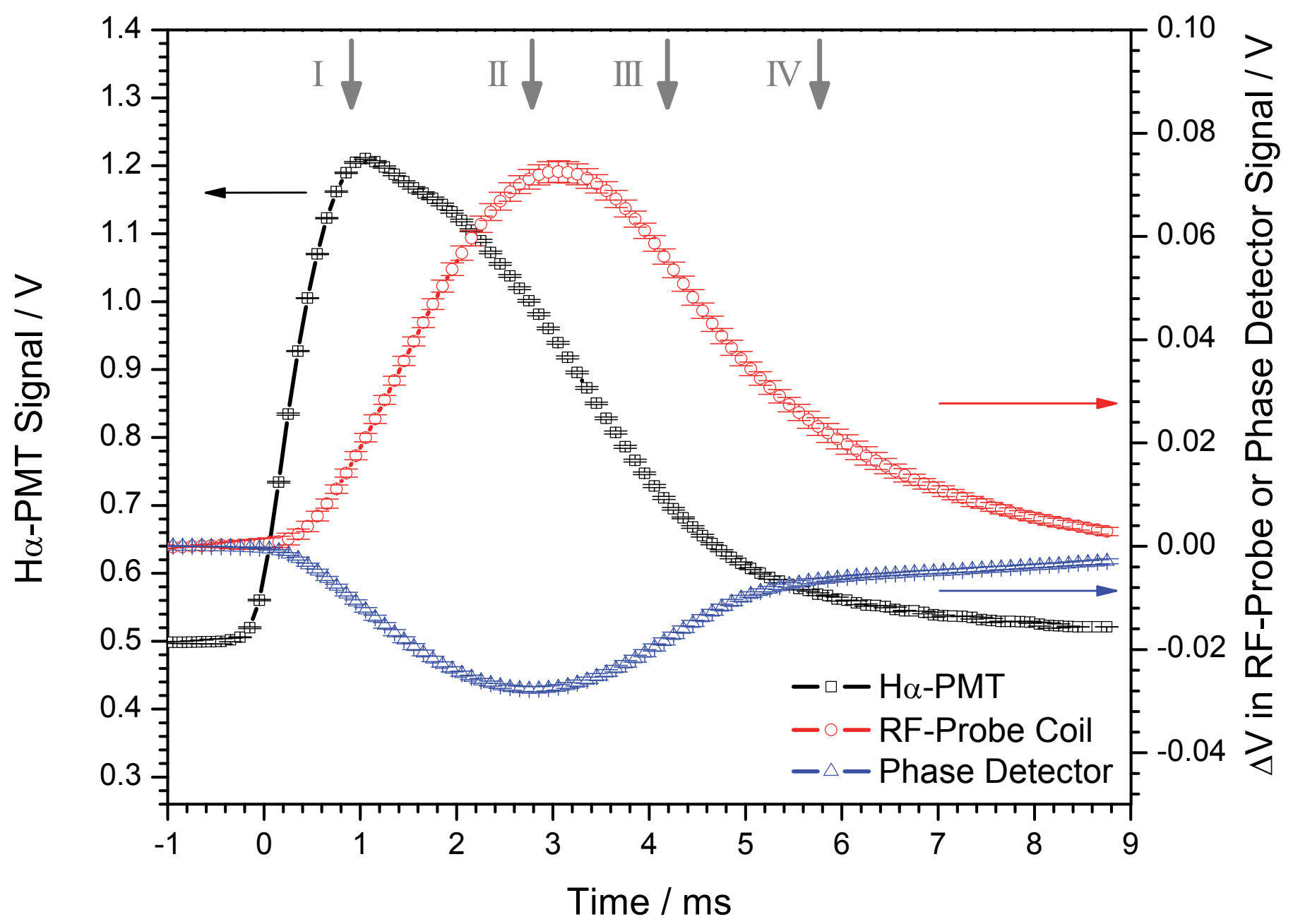


Figure 8b

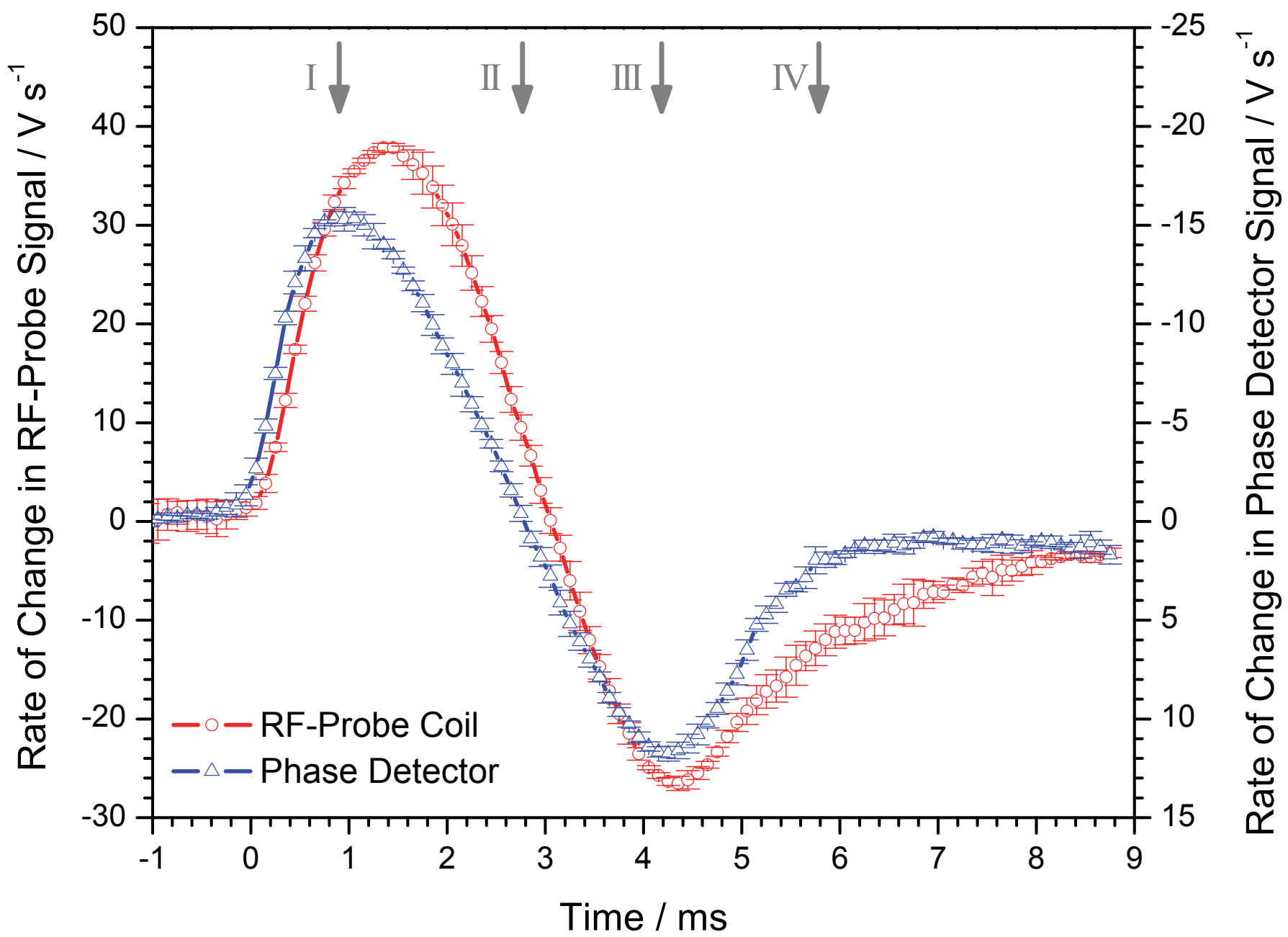


Figure 9

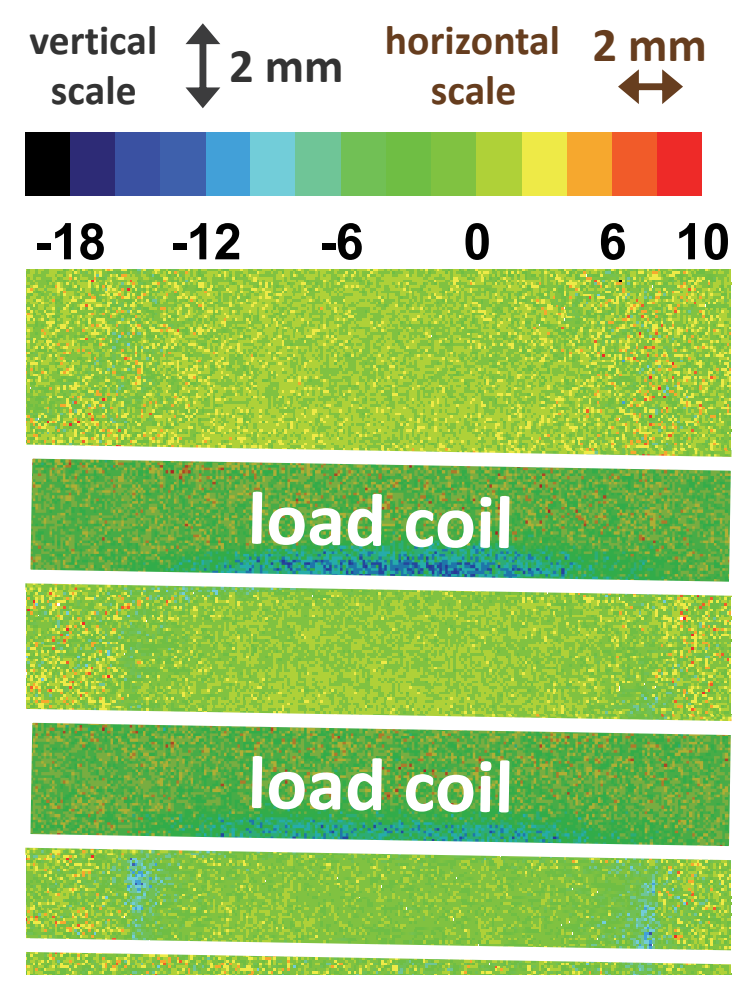

$0.45 \mathrm{~ms}$
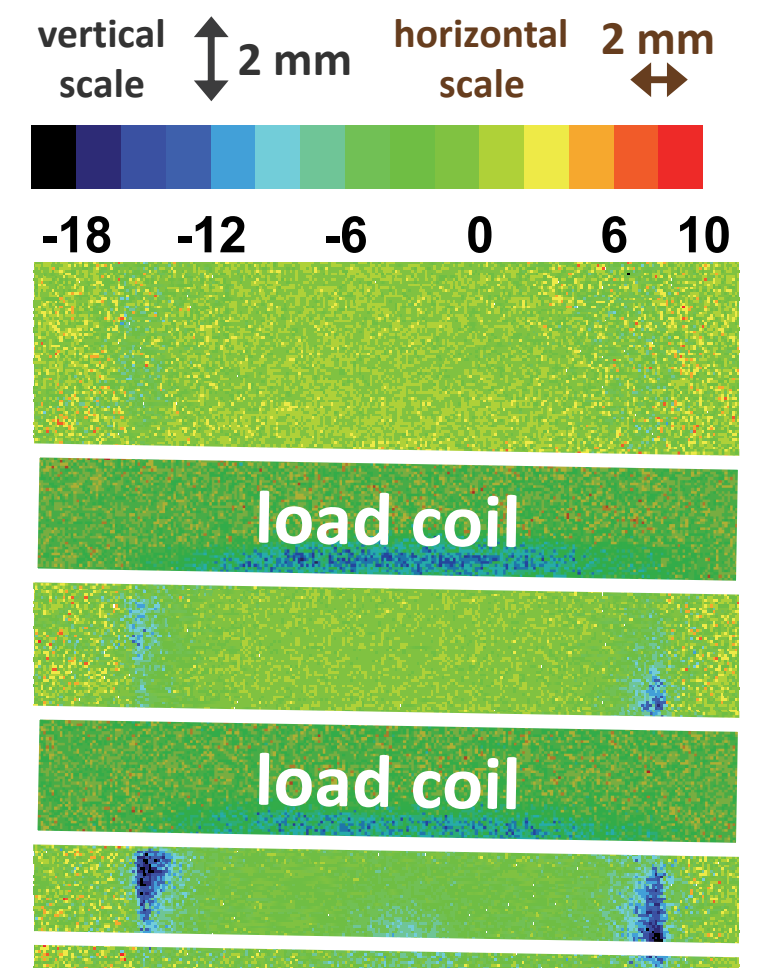

$0.75 \mathrm{~ms}$
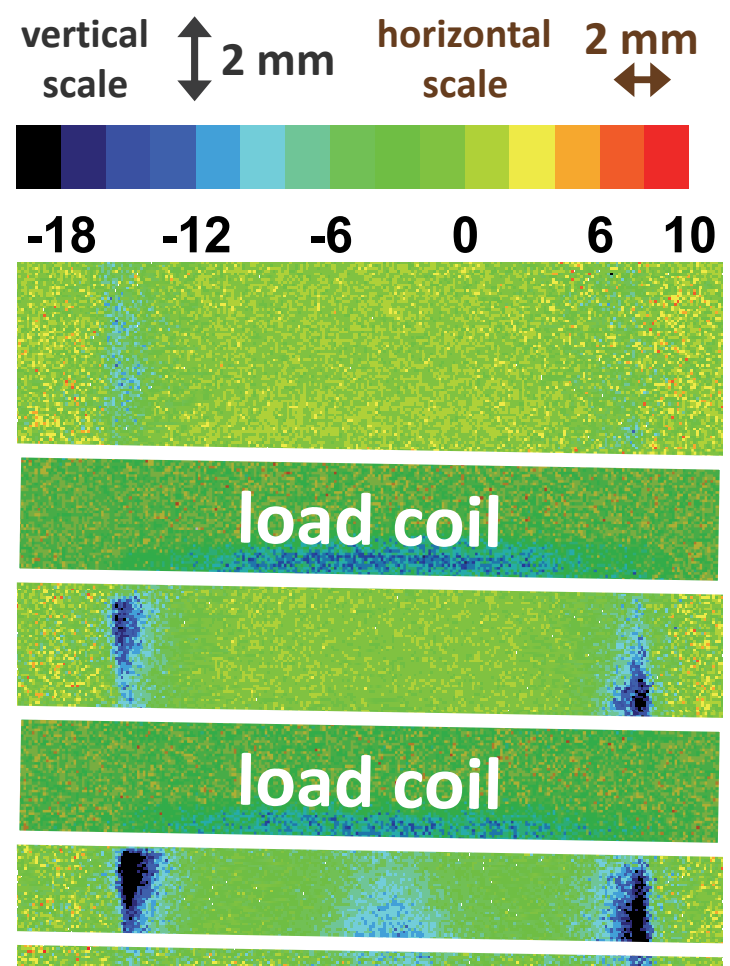

$1.05 \mathrm{~ms}$ 
Figure 10

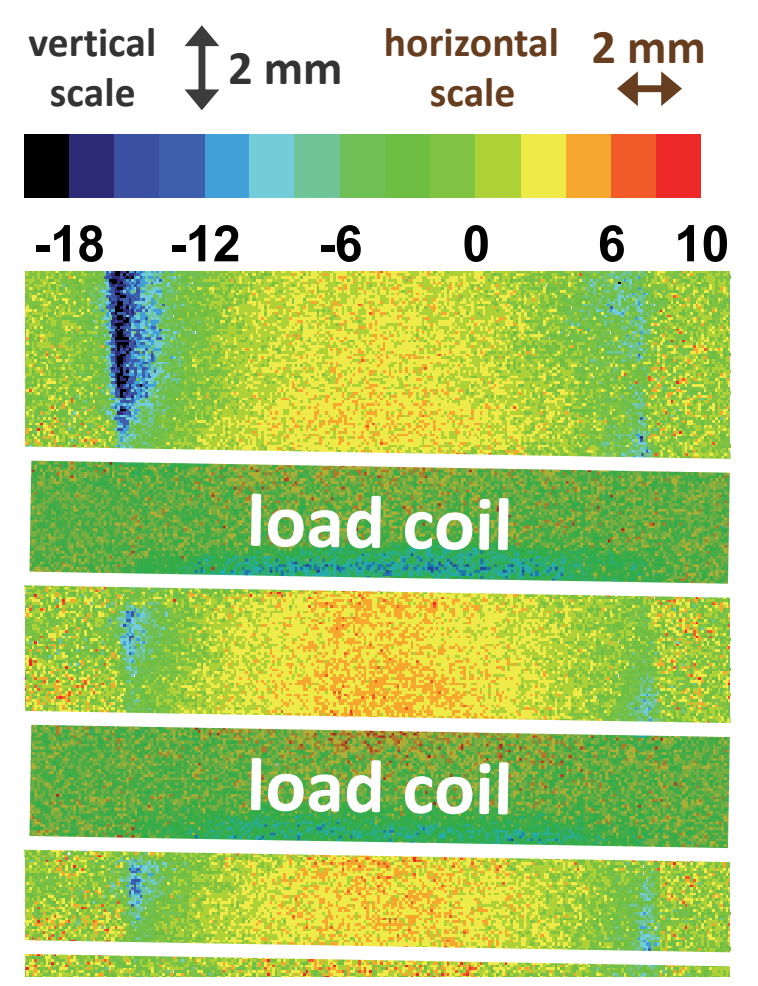

$5.15 \mathrm{~ms}$

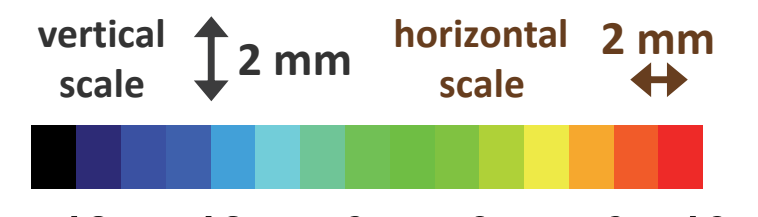

$\begin{array}{llllll}-18 & -12 & -6 & 0 & 6 & 10\end{array}$
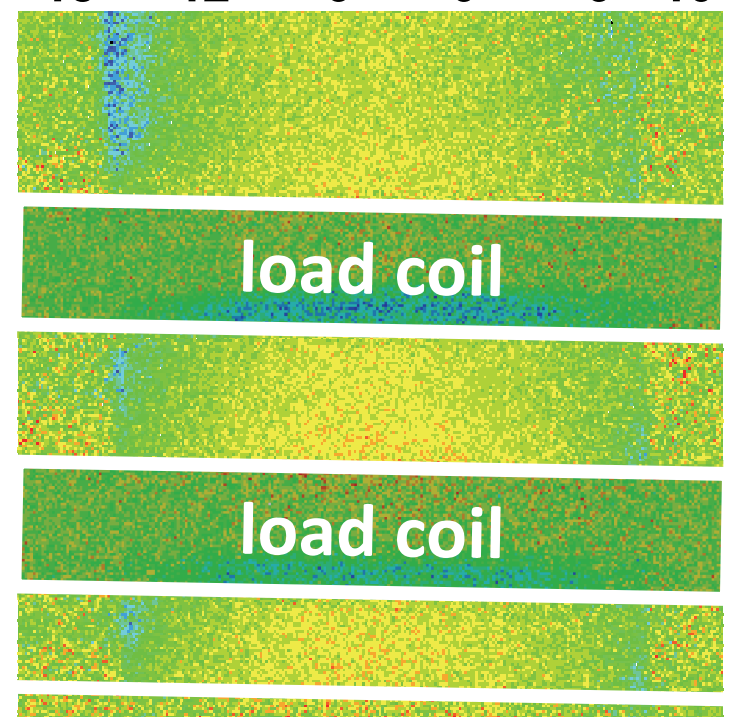

$5.75 \mathrm{~ms}$

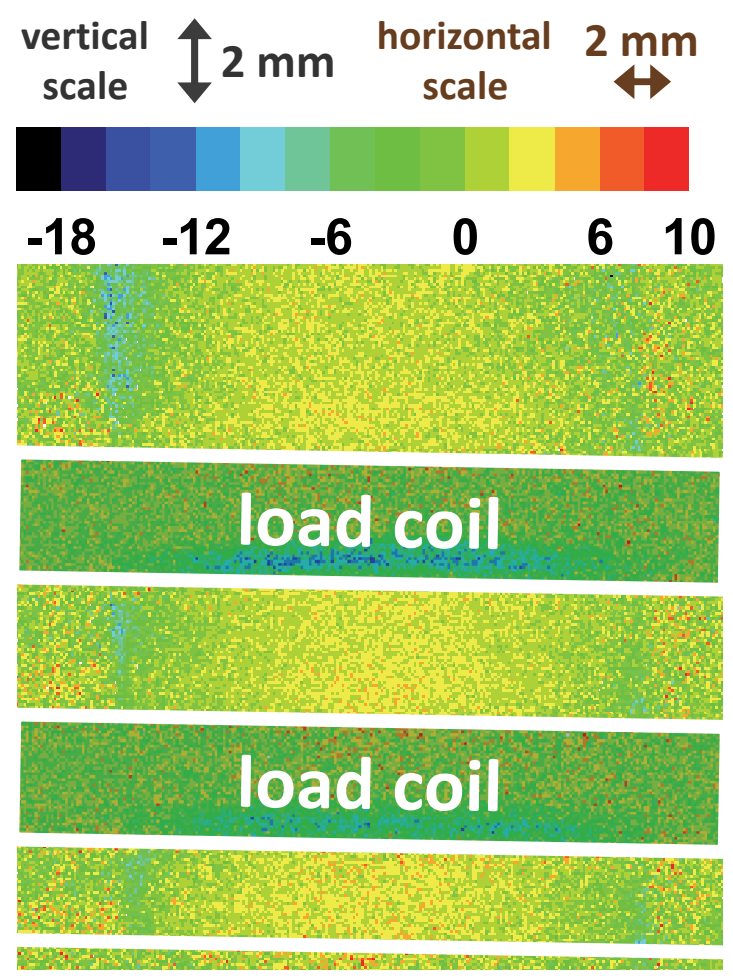

$6.35 \mathrm{~ms}$ 